\title{
Supernovae and Gamma-Ray Bursts
}

\author{
Philipp Podsiadlowski ${ }^{1}$ \\ Dept. of Astrophysics, University of Oxford, Oxford, OX1 3RH, UK
}

\begin{abstract}
Summary. Supernovae are explosions of stars which are triggered either by the implosion of the core of a star or a thermonuclear runaway, causing a bright optical display lasting for weeks to years. This chapter first explains the main explosion types, how they are classified and the principles that determine their lightcurves. It then discusses in more detail some of the most important supernova types, specifically SN 1987A, the last naked-eye supernova near our own Galaxy, Type Ia supernovae that have been used as standardizable cosmological distance candles, and gamma-ray bursts and their related supernovae. Special emphasis is given to the link of the various supernova types to their progenitor systems and a discussion of any outstanding issues. Causes for the large diversity of supernova types and sub-types are then systematically explored: these include binarity, the explosion mechanisms, rotation, metallicity and dynamical effects. Finally, some of the major topics of current interest are briefly discussed.
\end{abstract}

\section{Introduction}

Broadly speaking, a supernova is the explosion of a star or stellar system. It derives its name from a combination of the words nova, referring to a "new" star suddenly appearing in the sky, and super denoting a particularly bright "new" star. Novae and supernovae have been observed and described as important cosmic events for at least a few thousand years; the first recorded by the Chinese was SN 189 which occurred in 189 AD. The fact that novae and supernovae represent different physical phenomena was realized only in the 1930's by Baade \& Zwicky (1934). ${ }^{1}$ That these might involve the final collapse of the core of a massive star to a neutron star was proposed soon thereafter by Gamow \& Schönberg (1941), but it took until 1987 and the occurrence of supernova 1987A (SN 1987A) that this was finally confirmed observationally

\footnotetext{
${ }^{1}$ Unlike supernovae that generally involve the whole star in the explosion, novae are now understood to be thermonuclear explosions in the envelopes of white dwarfs.
} 
for at least one of the main supernova types. ${ }^{2}$ We now know that there are at least two major mechanisms producing a supernova, although in recent years with the discoveries of 100 s of new supernovae, it has become clear that there is an enormous diversity of supernova types and sub-types; there are almost certainly more than just two explosion mechanisms. In this chapter, we will first discuss the two major explosion mechanisms, the classical classification scheme and how a lot of the observed diversity can be understood relatively simply by variations of the envelope properties of the exploding stars. We will then discuss SN 1987A in some detail, the last naked-eye supernova which has been one of the major astronomical events of the 1980's and which has provided a major impetus in the supernova field, and the class of Type Ia supernovae, which ten years later provided the first evidence for an accelerating Universe. Gamma-ray bursts and related hypernovae, some of the largest explosions in the Universe, are the topic of the next section. This is followed by an overview over the various physical reasons that cause the observed diversity, and the chapter ends with a number of selected topics of particular current interest.

\section{Major Explosion Mechanisms}
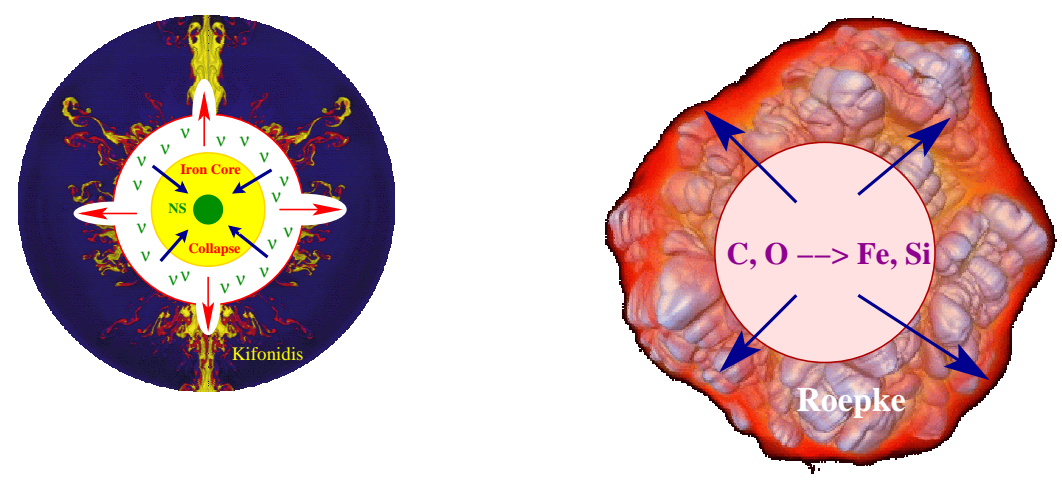

Fig. 1. Schematic figures contrasting a core-collapse supernova in the core of a massive star (left) to a thermonuclear explosion in a CO white dwarf (right).

\footnotetext{
2 Traditionally, supernovae are named after the year and the order in the year in which the supernova was reported; therefore, SN 1987A was the first supernova that was reported in 1987. Today, with the discovery of 100s of supernovae per year, not all supernovae are named based on this convention.
} 
For at least a few decades it has been realized that there are (at least) two main supernova explosion mechanisms: core-collapse supernovae involving the final phase in the evolution of a massive star and thermonuclear explosions, most likely related to white dwarfs approaching the Chandrasekhar limit. ${ }^{3}$

\subsection{Core-Collapse Supernovae}

The evolution of stars and, in particular, massive stars is characterized by an alternation of nuclear burning phases and contraction phases. For example, the Sun is presently burning hydrogen in its core at a temperature of $\sim 10^{7} \mathrm{~K}$. After it has consumed all of its hydrogen in the core, the core will start to contract and heat up until ultimately helium will ignite (at a characteristic temperature of $\sim 10^{8} \mathrm{~K}$ ). After helium burning, this cycle terminates for the Sun, as it developes a degenerate carbon/oxygen (CO) core and ends its evolution as a $\mathrm{CO}$ white dwarf. For significantly more massive stars $\left(M \gtrsim 11 M_{\odot}\right)$, the alternation of contraction and burning phases continues until ultimately the star has developed an iron core, surrounded by an onion-like structure consisting of shells of increasingly lower mean-atomic mass. Since iron is the most stable nucleus (i.e., has the highest nuclear binding energy per baryon), no more energy can be generated by fusing iron with other nuclei. Therefore, if the core exceeds the Chandrasekhar mass for iron, there is no longer a cold hydrostatic equilibrium configuration, and the core has to contract/collapse as it cools and loses its thermal-pressure support. While this contraction may start slowly, it soon accelerate because of a number of instabilities. At the end of silicon burning, the final nuclear burning phase producing the iron core, the central temperature and density of the core, $T_{\mathrm{c}}$ and $\rho_{\mathrm{c}}$, are $\sim 8 \times 10^{9} \mathrm{~K}$ and $\sim 4 \times 10^{12} \mathrm{~kg} \mathrm{~m}^{-3}$, respectively. Once the core has contracted enough and reaches a temperature $T_{\mathrm{c}} \sim 10^{11} \mathrm{~K}$, the iron and other heavy nuclei start to be photodissociated into $\alpha$ particles $\left({ }^{4} \mathrm{He}\right)$ and neutrons:

$$
\gamma+{ }^{56} \mathrm{Fe} \rightleftharpoons 13 \alpha+4 \mathrm{n}-124 \mathrm{MeV} .
$$

This photodissociation involves endothermic reactions that require energy $(124 \mathrm{MeV})$; as a consequence the temperature increases less rapidly than pressure, accelerating the contraction. At a slightly higher temperature $\left(T_{\mathrm{c}} \sim\right.$ $2 \times 10^{11} \mathrm{~K}$ ), the $\alpha$ particles themselves are photodisintegrated into protons and neutrons:

$$
\gamma+{ }^{4} \mathrm{He} \rightleftharpoons 2 \mathrm{p}+2 \mathrm{n}-28 \mathrm{MeV} .
$$

the maximization of entropy favours the right-hand sides (because of the larger number of particles), in the final state of statistical equilibrium the core will consist mainly of protons and neutrons. Note also that these reactions undo

\footnotetext{
3 The Chandrasekhar limit defines the maximum mass at which a zero-temperature, self-gravitating object can be supported by electron degeneracty pressure. For typical white dwarf compositions, this mass is close to $1.4 M_{\odot}$.
} 
all of the nuclear fusion reactions of the previous nuclear burning phases. This is possible, since the gravitational energy that is released in this phase corresponds to roughly $10 \%$ of the rest-mass energy of the core $\left(\sim 3 \times 10^{46} \mathrm{~J}\right)$, which exceeds the total efficiency of nuclear burning from $\mathrm{H}$ to $\mathrm{Fe}$ by more than a factor of 10 . In addition to these reactions, the material becomes increasingly neutron rich (core neutronization) due to electron captures such as

$$
\begin{gathered}
\mathrm{e}^{-}+(\mathrm{Z}, \mathrm{A}) \rightarrow \nu_{\mathrm{e}}+(\mathrm{Z}-1, \mathrm{~A}), \\
\mathrm{e}^{-}+\mathrm{p} \rightarrow \nu_{\mathrm{e}}+\mathrm{n},
\end{gathered}
$$

where $\mathrm{Z}$ and $\mathrm{A}$ refer to the charge and atomic number of a nucleus. Since these reactions take away electrons that provide an important pressure support, this further accelerates the contraction, helping to turn the initial contraction rapidly into a free-fall collapse. Most of the energy that is released in the collapse is ultimately converted into neutrinos (by the electron-capture processes listed above and other thermal neutrino processes), which freely escape from the core, at least initially.

This collapse is only stopped once matter reaches nuclear densities $\left(\rho_{\text {nuc }}\right)$ and the strong force becomes important, providing a sudden repulsive force. Because of the initial overcompression of the matter, now mainly composed of neutrons, the core bounces and drives an outward moving shock into the still infalling outer core. It was once hoped that this shock, which initially carries an energy of $\sim 10^{44} \mathrm{~J}$ could reverse the infall of the outer core and cause an outflow, i.e., drive a prompt explosion. But because of the continued photodisintegration of the infalling material, which requires $\sim 10^{44} \mathrm{~J}$ for $0.1 M_{\odot}$ of $\mathrm{Fe}$, this energy is quickly consumed; a prompt shock is always found to stall and is unable to drive an explosion.

The total energy that is released in the collapse is of order the binding energy of the neutron star forming at the centre $\left(G M_{\mathrm{NS}}^{2} / R_{\mathrm{NS}} \sim 3 \times 10^{46} \mathrm{~J} \simeq\right.$ $0.1 M_{\mathrm{NS}} c^{2}$ for $M_{\mathrm{NS}} \simeq 1.4 M_{\odot}$ and $\left.R_{\mathrm{NS}} \simeq 10 \mathrm{~km}\right)$. This is several orders of magnitude more than the binding energy of the outer core $\left(E_{\text {core }} \simeq 10^{44} \mathrm{~J}\right)$. However, most of this energy escapes freely in the form of neutrinos that only interact weakly with matter. It has remained one of the most enduring unsolved problems in supernova physics, how a fraction $(\sim 1 \%)$ of this energy can be deposited just below the accretion shock and be allowed to accumulate till enough energy is available to drive an explosion. In the presently favoured model of delayed neutrino-driven explosions, this may require more than $500 \mathrm{~ms}$, which is extremely long compared to the dynamical timescale of the proto-neutron star $(\sim 1 \mathrm{~ms})$. If this mechanism fails, matter will continue to fall onto the proto-neutron star and ultimately convert it into a black hole. ${ }^{4}$

\footnotetext{
${ }^{4}$ There are other ideas of how to generate a supernova explosion, e.g., involving jet-driven explosions, or very strong magnetic fields. The latter also requires a very rapidly rotating pre-supernova core. While present pre-supernova models do not predict sufficiently rapidly rotating cores, there may be special circumstances
} 


\subsection{Thermonuclear Explosions}

The second important explosion mechanism has nothing to do with massive stars, but is generally believed to occur in accreting $\mathrm{CO}$ white dwarfs when their mass approaches the Chandrasekhar mass. When the mass reaches $\sim 1.37 M_{\odot}$, carbon is ignited in or near the centre of the white dwarf. Initially, this drives convection in the core, transporting the energy outwards and radiating it away in the form of neutrinos (this phase of low-level carbon burning, referred to as the simmering phase, can last for up to $\sim 10^{3} \mathrm{yr}$ ). But, there comes a point when the core is unable to rid itself of the excess nuclear energy, and the burning process becomes explosive. The reason for this nuclear runaway is that the core material is highly degenerate. This means that the core pressure is independent of temperature. Therefore, a rise in central temperature (due to the carbon burning) does not produce an increase in pressure which would limit the increase in temperature (the valve mechanism that keeps burning in ordinary stars, supported by thermal pressure, stable). The further increase in temperature increases the nuclear burning further, producing a runaway process which incinerates a large fraction of the white dwarf and ultimately destroys it completely. In the case of a thermonuclear explosion, unlike the case of core collapse, no remnant is expected, and the energy source is purely nuclear energy $\left(\sim 10^{44} \mathrm{~J}\right)$. The fact that the energy in the two types of explosion is comparable $\left(\sim 10^{44} \mathrm{~J}\right)$ is not a coincidence, since, in both cases, the energy scale is set by the binding energy of the core (the $\mathrm{CO}$ core in the case of the thermonuclear explosion, and the binding energy of the outer Fe core in the core-collapse case), which are comparable (they are ultimately determined by the same physics of electron degeneracy, which determines the immediate pre-supernova structure).

In the ensuing explosion, a large fraction of the white dwarf is burned, in the inner part completely to nuclear statistical equilibrium (NSE), which means mainly to iron-group elements, mostly ${ }^{56} \mathrm{Ni}$, and incompletely further out, producing mainly intermediate-mass elements, such as ${ }^{28} \mathrm{Si}$ and ${ }^{32} \mathrm{~S}$. The radioactive ${ }^{56} \mathrm{Ni}$ will subsequently decay to ${ }^{56} \mathrm{Co}$ (with a half-life of $6.1 \mathrm{~d}$ ), powering the supernova lightcurve, and ultimately to ${ }^{56} \mathrm{Fe}$ (with a half-life of $77.3 \mathrm{~d}$ ). A typical supernova of this type produces $\sim 0.7 M_{\odot}$ of ${ }^{56} \mathrm{Fe}$; hence these supernovae are believed to be the dominant producers of iron in the Universe. Since most of them produce very similar amounts of radioactive ${ }^{56} \mathrm{Ni}$, the resulting supernova lightcurves are quite similar, which means that they can be used as standard distance candles (strictly speaking 'standardizable' distance candles; see $§ 5.1$ ).

Unlike core-collapse supernovae, the physics of thermonuclear explosions is reasonably well understood. One of the lingering uncertainties is how the carbon burning front, which starts as a deflagration (i.e., a sub-sonic burning front), is accelerated into a detonation (i.e., a super-sonic burning front),

in which this is the case, and this may be the origin of magnetars, neutron stars with very large magnetic fields, or even gamma-ray bursts (see $\S 7.2$ ). 
which seems to be favoured by observations for the majority of thermonuclear explosions.

The main uncertainty, even controversy, is the question of their progenitors, the type of stellar systems in which a $\mathrm{CO}$ white dwarf can grow towards the Chandrasekhar mass. We will return to this issue in $\S 5.2$.

\section{Supernova Classification}

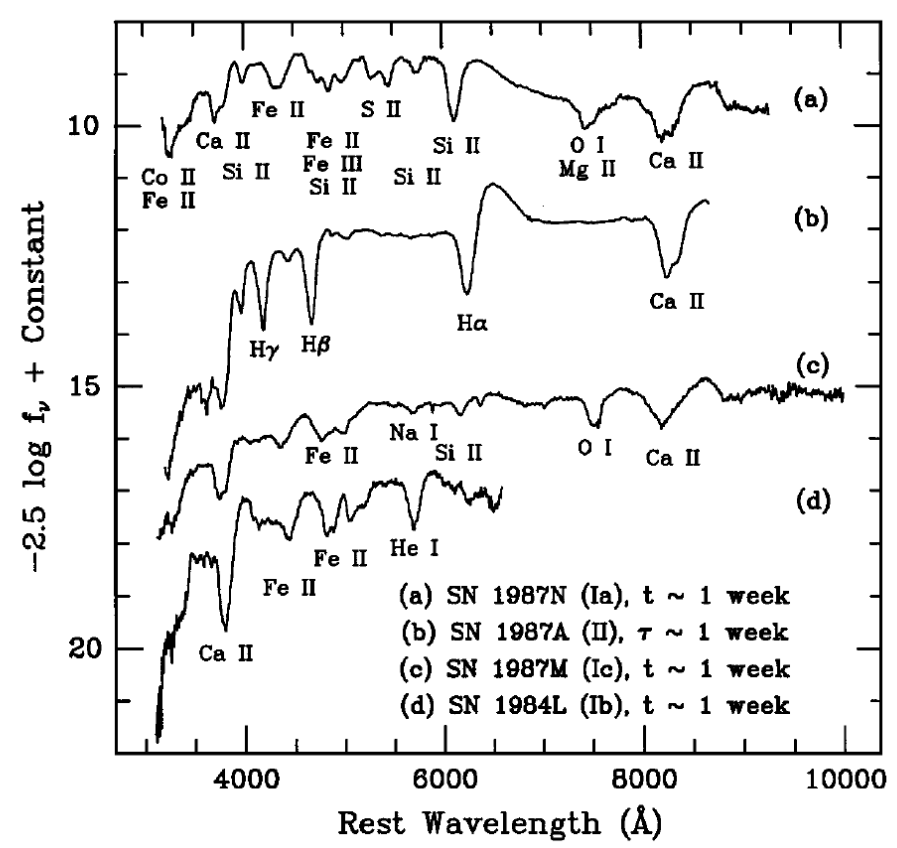

Fig. 2. SN Spectral Classification. SN Ia (a), SN II (b), SN Ic (c), SN Ib (d). (From Filippenko 1997.)

The basic classification of supernovae is quite simple: they are classified as Type I or Type II supernovae, depending on whether they have hydrogen lines in the spectrum (Type II) or lack hydrogen lines (Type I). For a long time, it was thought that these two observational classes may have a one-toone relation to the two explosion mechanisms discussed in the last section, core collapse supernovae (Type II) and thermonuclear explosions (Type I), respectively. However, over the last three decades is has become clear that this is not the case and that, in principle, both explosion types could come in both observational varieties. As a consequence, the basic classification has 
become much more complex, requiring the introduction of more and more sub-types.

\subsection{Main Classification Scheme}

\begin{tabular}{|c|c|c|c|c|c|}
\hline \multicolumn{3}{|c|}{$\begin{array}{c}\text { Type I } \\
\text { no H }\end{array}$} & \multicolumn{3}{|c|}{$\begin{array}{c}\text { Type II } \\
\text { H }\end{array}$} \\
\hline $\begin{array}{c}\mathrm{Ia} \\
\mathrm{Si}(\mathrm{S}) \text { lines }\end{array}$ & $\begin{array}{c}\mathrm{Ib} \\
\text { no } \mathrm{Si}, \text { but } \mathrm{He}\end{array}$ & $\begin{array}{c}\text { Ic } \\
\text { no } \mathrm{Si}, \text { no } \mathrm{He}\end{array}$ & $\begin{array}{c}\text { II-P } \\
\text { plateau } \\
\text { lightcurve }\end{array}$ & $\begin{array}{c}\text { II-L } \\
\text { linear } \\
\text { lightcurve }\end{array}$ & $\begin{array}{c}\text { IIb } \\
\text { change type } \\
\text { II } \rightarrow \text { Ib }\end{array}$ \\
\hline thermonuclea & & core & collapse & & \\
\hline
\end{tabular}

Table 1. Supernova Spectral Classification Scheme.

The thermonuclear explosion of a CO white dwarf is now believed to be associated with a Type Ia supernova (SN Ia). These supernovae have no hydrogen, but strong Si lines. Si and also S are intermediate-mass nuclei which are produced in abundance in the part of the exploding white dwarf that does not burn completely to NSE, and therefore provides a very characteristic signature for a thermonuclear explosion.

In addition to SNe Ia, there are two other sub-types of Type I supernovae, Type Ib and Type Ic. These types are also defined on the basis of their spectroscopic characteristics, both lack hydrogen, but Type Ib supernovae (SNe Ib) show He lines, while Type Ic supernovae (SNe Ic) lack both $\mathrm{H}$ and He lines. Unlike SNe Ia, they produce fairly little ${ }^{56} \mathrm{Ni}$ and are found predominantly in or near star-forming regions and are therefore believed to be connected with core-collapse supernovae, i.e., the explosions of massive stars that have lost their H-rich envelopes and, in the case of SNe Ic, their He-rich layers as well. ${ }^{5}$

There are also several different sub-types of Type I supernovae. Unlike SNe I, they are not always defined by their spectroscopic properties but by their lightcurves, i.e., their luminosity, measured in a particular waveband (typically $B$ or $V$ ) as a function of time. The lightcurves of Type II-P supernovae (SNe II-P), where the 'P' stands for "plateau", show a long phase, lasting up to $\mathrm{a} \sim 100 \mathrm{~d}$ where the lightcurve is nearly constant (the plateau phase). Their progenitors are most likely massive red supergiants (with a typical mass $\lesssim 20 M_{\odot}$ ) that experience core collapse. The second, much less common variety, Type II-L supernovae (SNe II-L), do not show this plateau but drop off

\footnotetext{
${ }^{5}$ It is presently not entirely clear how much He could be present in a SN Ic. Since $\mathrm{He}$ is non-thermally excited, it requires the presence of a source of energetic photons, e.g., from the radioactive decay of ${ }^{56} \mathrm{Ni}$. If the He layer is shielded from this radioactive source, it is possible in principle to hide significant amounts of He. However, the most recent estimates (Hachinger et al. 2012) suggest that at most $0.2 M_{\odot}$ can be hidden.
} 
more or less linearly (on a logarithmic scale) after their lightcurve has peaked (hence the letter ' $L$ ' for "linear"). These are almost certainly also core-collapse supernovae, but in this case, the progenitors must have already lost a large fraction of their H-rich envelopes.

\subsection{Complications}

Unfortunately, there are many further complications going beyond this simple scheme. The progenitor of supernova 1987A (SN 1987A, see $\S 4$ ) had a large $\mathrm{H}$-rich envelope, but did not have an extended plateau phase (see Fig. 3) and therefore SN 1987A defines a class of its own. Other supernovae appear to change their type. Supernova 1993J initially looked like a type II supernova, but soon transformed into a supernova resembling a SN Ib. As a consequence, this supernova type is now referred to as a Type IIb supernova (SN IIb). Other sub-types are not directly related to a particular supernova mechanism, but to a supernova-related phenomenon. For example, Type IIn supernovae stand for supernovae that show narrow $\mathrm{H}$ lines $(\mathrm{H} \alpha)$ in emission. These must come from H-rich material in the immediate neighborhood of the supernova, most likely ejected by the progenitor in the not-too-distant past, that was flashionized by the first light from the supernova. This is not necessarily related to a particular explosion type; it just implies a particular mass-loss history of the progenitor. In a more extreme version, there may be so much material around the exploding star that the supernova ejecta are rapidly slowed down by the interaction with this material, converting kinetic energy into thermal energy and ultimately radiation. In this case, the lightcurve shape itself is determined by this interaction with the circumstellar material. Supernovae (e.g., $\S 8.3$ ) that show evidence for such interactions are sometimes referred to as Type IIa supernovae (SNe IIa), though how this fits into the overall supernova scheme and, in particular, its relation to SNe IIb devies any obvious logic.

Indeed, as this previous discussion shows, the supernova classification scheme has become too complicated and convoluted to be very useful. In fact, sometimes even supernova experts get confused. The problem is that the main scheme is a discrete one, while the supernova properties clearly vary in a continuous manner. What one would like from a theoretical point of view is a scheme that first specifies the explosion mechanism and then has one or more continuous parameters that relate to the key properties of the progenitor that vary from supernova to supernova (such as the envelope mass; see $\S 3.3$ ). In addition, one needs a parameter that describes the environment in which the supernova occurs, in particular, the circumstellar material that may be a direct result of the mass-loss history of the progenitor.

\subsection{The Diversity of Core-Collapse Supernova Lightcurves}

An observable supernova requires the ejection of material (the envelope of a collapsing star or a completely disrupted star) that cools and radiates away 
its energy. In general there are two main sources for the observed radiation: one is the shock energy that was deposited in the ejecta by the initial shock wave. The second is radioactive energy from the decay of radioactive ${ }^{56} \mathrm{Ni}$ that is produced in the explosion itself that decays first to ${ }^{56} \mathrm{Co}$ (with a half-life of $6.1 \mathrm{~d}$ ) and then to ${ }^{56} \mathrm{Fe}$ (with a half-life of $77.3 \mathrm{~d}$ ). In a Type II supernova the main lightcurve is generally dominated by the release of shock energy. It is only at late times that the lightcurves are powered by radioactivity, leading to a characteristic exponential lightcurve tail. The latter allows an estimate for the amount of ${ }^{56} \mathrm{Ni}$ that was produced in the explosion. In the case of Type I supernovae, which generally have very compact progenitors, the shock energy escapes very quickly and is generally not observed, and the main lightcurve peak is powered by radioactivity. In both cases, the width of the main peak of the lightcurve is determined by the diffusion timescale on which photons escape from the expanding ejecta.

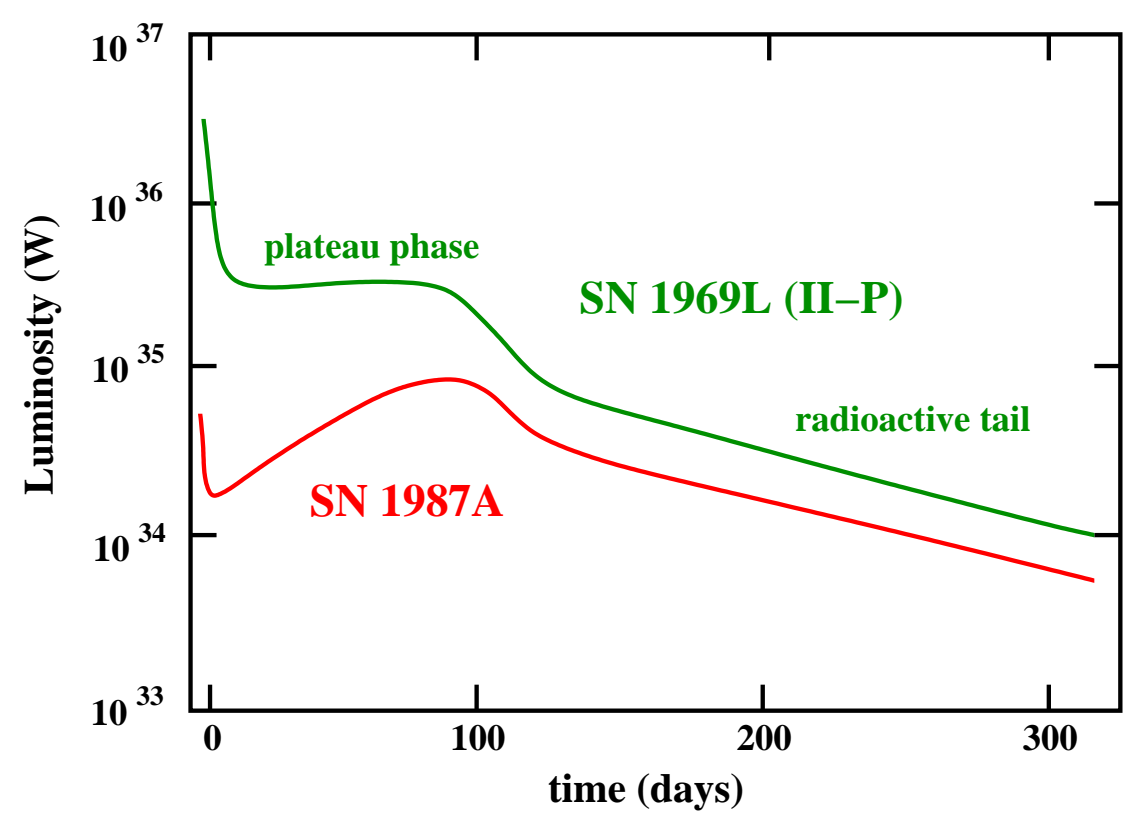

Fig. 3. Bolometric supernova lightcurves for two Type II supernovae. In both cases, the progenitor was a star with a massive hydrogen envelope, but in the case of SN 1969L the progenitor was a red supergiant, while for SN 1987A it was a blue supergiant with $R \simeq 40 R_{\odot}$.

Even though there is an enormous diversity of supernova lightcurves of core-collapse supernovae, a lot of this diversity is relatively easy to understand as a variation of the envelope properties, specifically its radius and mass, even if the explosions at the centre are identical. Fig. 3 compares the lightcurve of 
$\odot$

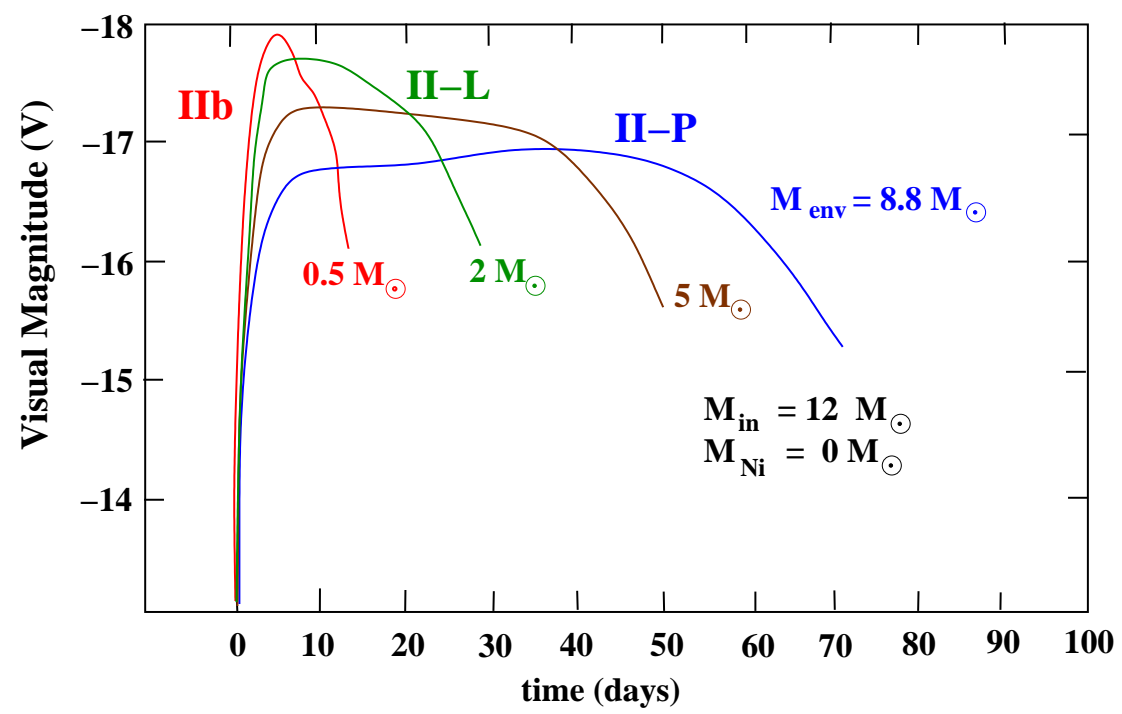

Fig. 4. Theoretical visual lightcurves for red-supergiant progenitors with varying masses of hydrogen (as indicated) in the red-supergiant envelope, illustrating the dependence of the lightcurves on envelope mass. (Adapted from Hsu [1991].)

a typical SN II-P (SN 1969L) to the lightcurve of SN 1987A. Both supernovae probably had massive progenitors with large H-rich envelopes. But the difference is that the progenitor of SN 1969L almost certainly was a red supergiant, with a radius of $\sim 1000-2000 R_{\odot}$, while the progenitor of SN 1987A only had a radius of $\sim 40 R_{\odot}$. In the latter case, a significant fraction of the explosion energy was consumed in expanding the ejecta from its more compact, more tightly bound configuration, making it less luminous, at least initially.

An even more important parameter is the envelope mass. This is illustrated in Fig. 4 which shows theoretical lightcurves (in this case, $V$-band magnitude versus time) for a star of initially $12 M_{\odot}$ that was assumed to have lost different amounts of envelope mass before the explosion (in all cases, the star is a red supergiant at the time of explosion). All simulations start by depositing the same amount of explosion energy $\left(10^{44} \mathrm{~J}\right)$ in the centre. For the case, where the star has lost none of its envelope and has a final presupernova H-rich envelope mass of $8.8 M_{\odot}$ (as labelled), the lightcurve is that of a typical SN II-P with a plateau phase lasting $\sim 50 \mathrm{~d}^{6}$ As the envelope mass

\footnotetext{
${ }^{6}$ The reason why this lightcurve does not show the initial peak seen for SN 1969L in Fig. 3 is that Fig. 3 shows a bolometric lightcurve, while Fig. 4 shows a $V$-band lightcurve. The $V$-band lightcurve peaks later, as initially most of the radiation from the supernova escapes in the UV.
} 
is reduced, the plateau shortens and the overall luminosity increases (as the total energy input is the same). Once the envelope mass has dropped below $2 M_{\odot}$, the plateau has disappeared and this lightcurve would be classified as a SN II-L. As the envelope mass is reduced further to $0.5 M_{\odot}$, the lightcurve only has a short peak where hydrogen would be visible in the spectrum; this would classified as a SN IIb. If the hydrogen envelope is lost completely, one would have a SN Ib and, if the He layer is lost as well, a SN Ic. Thus, the whole sequence,

$$
\mathrm{SN} \mathrm{II-P} \rightarrow \mathrm{SN} \mathrm{II-L} \rightarrow \mathrm{SN} \mathrm{IIb} \rightarrow \mathrm{SN} \mathrm{Ib} \rightarrow \mathrm{SN} \text { Ic }
$$

is just a sequence of increased envelope loss, first of the H-rich envelope and then the He-rich layer. The immediate physical question is what causes this mass loss. While stellar winds may play an important role in some cases, binary interactions are almost certainly even more important, since a large fraction, if not the majority, of all massive stars are in relatively close binaries where the components can interact directly (e.g., by mass transfer causing mass loss, mass accretion and, in the most extreme case, by the complete merger of the binary components [Podsiadlowski, Joss \& Hsu 1992]; see $\S$ 7.1). These interactions particularly affect the envelope properties of the massive progenitors and hence help to determine the shapes of the resulting lightcurves.

\section{Understanding SN II-P lightcurves}

Most of the key properties of a II-P lightcurve are relatively easy to understand from basic principles. A supernova is basically a huge explosion where a certain amount of energy, the explosion energy $\left(E_{\text {exp }}\right)$, is suddenly put in the centre of a large stellar envelope. The explosion energy is determined by the details of the explosion mechanism; in the presently favoured delayed neutrino-driven explosion, this energy accumulates till it is sufficient to unbind the parts of the core that do not become part of the central neutron star; this sets a natural energy scale for $E_{\exp } \sim 10^{44} \mathrm{~J}$. Since this energy is injected on a timescale of $\lesssim 1 \mathrm{~s}$, which is short compared to the dynamical timescale of even the core of the star, this will drive a shock front, propagating outwards through the rest of star and accelerating it in the process. The first visible sign of the supernova (apart from the neutrino signal from the initial collapse that propagates with the speed of light) appears when the shock reaches the photosphere which finds itself suddenly heated to $0.5-1 \times 10^{6} \mathrm{~K}$, the temperature behind the shock. ${ }^{7}$ From this point onwards, the supernova can be considered an expanding and cooling sphere of gas. It is luminosity is determined

\footnotetext{
${ }^{7}$ Even before the shock reaches the surface of the star, some radiation generated in the shocked region will diffuse outwards faster than the shock front itself moves; when this radiation escapes from the progenitor it produces a radiative precursor which precedes the actual shock breakout. Such a precursor has been observed for the II-P supernova SNLS-04D2dc (Schawinski et al. 2008).
} 
by the location of the photosphere, ${ }^{8}$ which recedes in mass coordinates, but may either expand or contract in radius coordinates, and the characteristic temperature at the photosphere. Since this temperature is initially very high $\left(\sim 10^{6} \mathrm{~K}\right)$, the spectrum will first peak in soft X-rays and the UV (producing a short-lived $U V$ flash that will ionize any nearby circumstellar material), moving steadily towards the B and V, as the ejecta cool, mainly due to adiabatic expansion.

The supernova shock accelerates as it moves through the envelope towards lower-density regions. As a result, the ejecta reach their highest velocities in the very outermost layers (this velocity may easily exceed $0.1 c$, where $c$ is the speed of light). The characteristic velocity of the ejecta on the whole can be estimated simply from energy conservation,

$$
E_{\mathrm{exp}} \sim \frac{1}{2} M_{\mathrm{ej}} v_{\mathrm{ej}}^{2}
$$

where $M_{\text {ej }}$ and $v_{\text {ej }}$ are the mass and characteristic velocity of the ejecta, respectively (this ignores the binding energy of the envelope which is not significant for red supergiants). For typical values, this yields

$$
v_{\mathrm{ej}} \sim 3000\left(\frac{E_{\mathrm{exp}}}{10^{44} \mathrm{~J}}\right)^{1 / 2}\left(\frac{M_{\mathrm{ej}}}{10 M_{\odot}}\right)^{-1 / 2} \mathrm{~km} \mathrm{~s}^{-1} .
$$

As the energy that was deposited by the initial shock has to diffuse out of this expanding sphere of gas, the overall length of the lightcurve is determined by the diffusion time, which is given by

$$
t_{\mathrm{diff}}=\frac{R^{2}}{l c}
$$

where $R$ is the radius of the sphere and $l$ the mean free path of the diffusing photons (this formula can be derived from a simple random-walk process). The mean free path itself can be related to the opacity $\kappa$ and density $\rho$ of the ejecta according to $l=1 / \kappa \rho$. Approximating $\rho$ by $M_{\mathrm{ej}} / 4 R^{3}$, eq. (4) can be rewritten as

$$
t_{\mathrm{diff}} \sim \frac{M_{\mathrm{ej}} \kappa}{4 R c} .
$$

However, $R$ itself increases with time $t: R(t) \sim v_{\text {ej }} t$. Substituting this into eq. (5) and setting $t=t_{\text {diff }}$, one can solve the resulting equation for $t_{\text {diff }}$ to obtain

$$
t_{\text {diff }} \sim \frac{M_{\mathrm{ej}}^{3 / 4} \kappa^{1 / 2}}{2\left(2 E_{\mathrm{exp}}\right)^{1 / 4} c^{1 / 2}} \simeq 150 \mathrm{~d}
$$

(for $E_{\text {exp }}=10^{44} \mathrm{~J}, M_{\mathrm{ej}}=10 M_{\odot}, \kappa=0.034 \mathrm{~m}^{2} \mathrm{~kg}^{-1}$ for pure electron scattering in a solar-type plasma). This estimate is in reasonable agreement with

\footnotetext{
${ }^{8}$ Strictly speaking, there is no well defined photosphere, as the point where the optical depth is of order unity is a strong function of wavelength.
} 
detailed numerical calculations. The flat portion of the lightcurve in Fig. 3, the plateau phase, starts when the temperature at the photosphere has fallen sufficiently so that $\mathrm{H}$ starts to recombine (at a typical temperature $T \sim 6000 \mathrm{~K}$ ). Since the recombination releases the ionization energy of $\mathrm{H}$, this helps to keep the photosphere hotter than it otherwise would be (H recombination acts like a thermostat keeping the temperature at $\sim 6000 \mathrm{~K}$ ). Since the photospheric radius changes only slowly in this phase, even though the $\mathrm{H}$ recombination front moves inwards in mass coordinates, the overall luminosity remains roughly constant.

Once the photosphere has passed through the H-rich ejecta, the lightcurve tends to drop sharply and at late times shows the characteristic exponential tail due to the radioactive decay of ${ }^{56} \mathrm{Co}$, which itself is the decay produce of ${ }^{56} \mathrm{Ni}$, produced in the explosion.

\section{Supernova 1987A in the Large Magellanic Cloud}

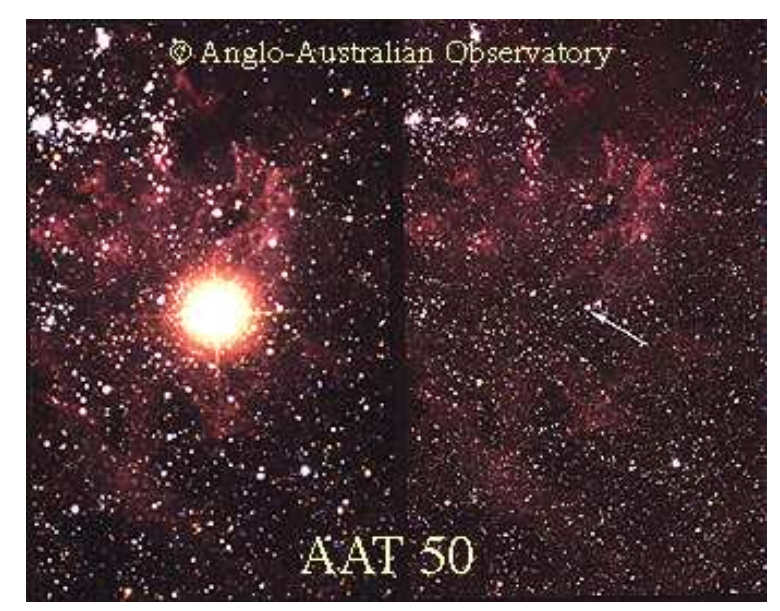

Fig. 5. Before and after images (right and left), taken with AAT of SN 1987A in the Large Magellanic Cloud. The arrow in the before picture points at the progenitor, a blue supergiant.

Supernova 1987A (SN 1987A) was the first supernova visible with the naked eye since Keplers supernova in 1604. It occurred on February 23, 1987 in the Large Magellanic Cloud, a satellite galaxy of our own galaxy, the Milky Way, about 180,000 lightyears away. Since it was the first nearby supernova seen in almost 400 years, it has long been awaited by astronomers and therefore was one of the major astronomical events of the $80 \mathrm{~s}$. The observations of neutrinos from the supernova confirmed that supernovae are produced by the collapse of a massive star, leading to the formation of a neutron star. 
The Mystery of the Progenitor Star
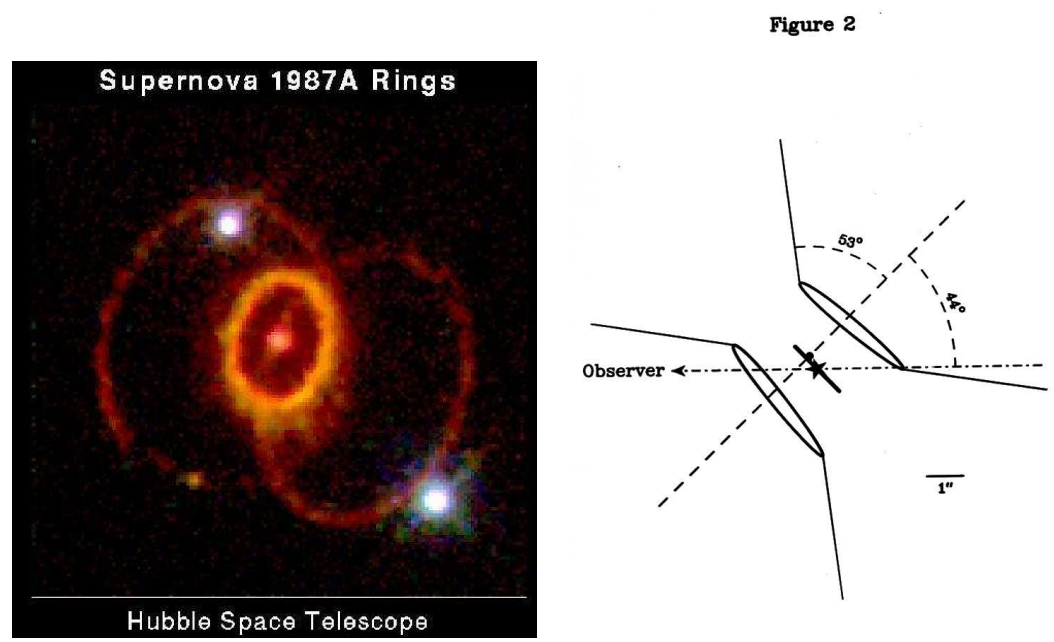

Fig. 6. The triple ring nebula around SN $1987 \mathrm{~A}$ as observed with the HST (left) and a geometric model of the three rings (right). The material in the three rings was ejected by the supernova progenitor $\gtrsim 20,000 \mathrm{yr}$ before the explosion.

While SN 1987A confirmed some long-held beliefs about supernovae, the star that exploded defied expectations and provided a major mystery. Images of the region taken before the explosion (Fig. 5) showed that the star that exploded was a blue supergiant with an initial mass around $20 M_{\odot}$ and a radius at the time of the explosion of $40 R_{\odot}$. Theoretical models of the evolution of massive stars had predicted that these end their evolution as red supergiants with a radius at least $10^{3} R_{\odot}$. In addition, the composition of the star that exploded was very unusual; most importantly, the outer layers of the star had an abundance of helium (as a fraction of the total composition) that was about a factor of 2 larger than the expected abundance, as if part of the material from the core, where helium has been produced during the previous evolution of the star, was mixed into the outer layers by some unexpected mixing process. Why was the star blue and not red and why were there these chemical anomalies?

A major clue to resolve this mystery was the discovery of the triple-ring nebula around the supernova (Burrows et al. 1995). The nebula is composed of three overlapping rings, seen in projection (see the left panel of Fig. 6), and consists of material that was ejected from the progenitor star some 20,000 years before the explosion. The supernova occurred right at the centre of the inner ring, while the outer rings lie in planes below and above the central ring plane. The whole nebula was flash-ionized by the supernova, and we are now 
seeing the afterglow of this flash. The nebula is almost axisymmetric. This generally suggests that rotation may have played an important role in the shaping of the nebula. However, any star that was rapidly rotating early in its evolution could not have been rapidly rotating as a supergiant. This essentially ruled out that the progenitor star could have been a normal single star and strongly suggested that the progenitor was a member of a binary system, specifically a system where the two binary components merged completely $20,000 \mathrm{yr}$ before the explosion.

\section{A Binary Merger Model for the Progenitor of SN 1987A}

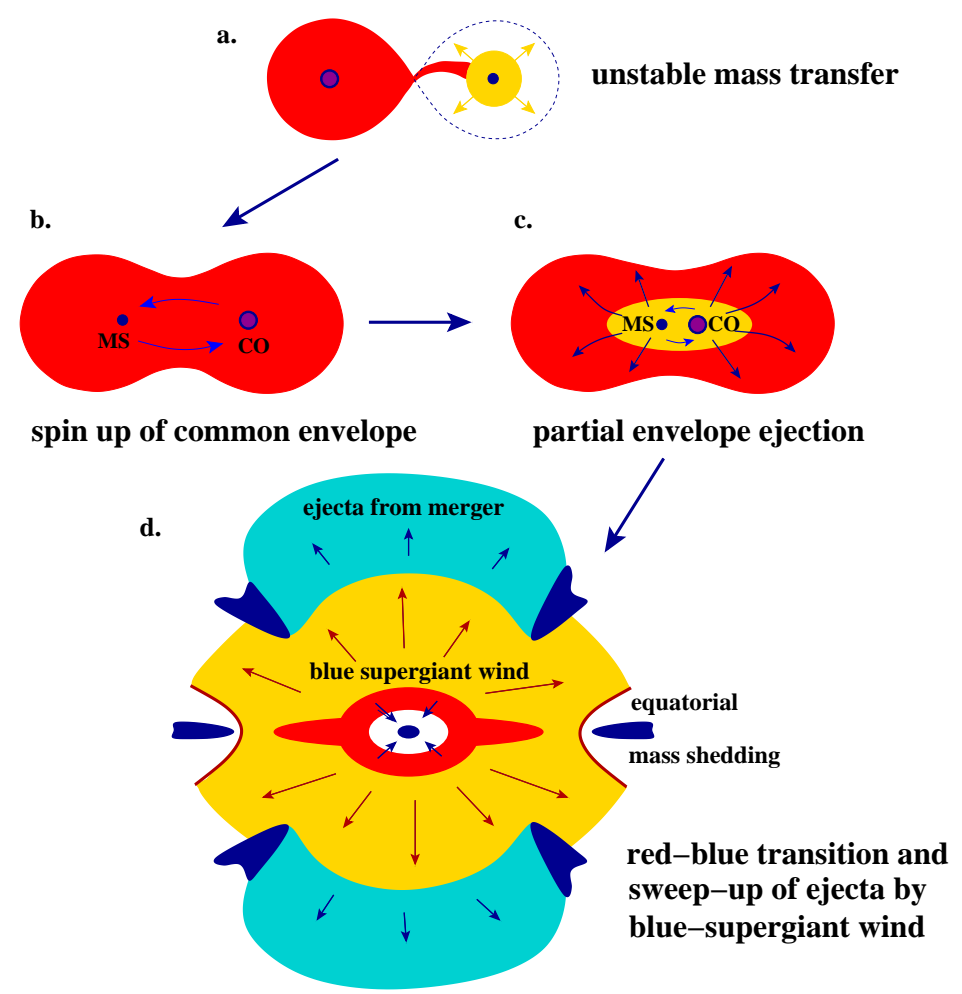

Fig. 7. Cartoon illustrating the probable evolution of the SN 1987A progenitor and the formation of the triple-ring by a merger $\sim 20,000 \mathrm{yr}$ before the explosion (from Morris \& Podsiadlowski 2007).

The idea that the anomalous properties of the progenitor of SN 1987A are the result of the merger had already been proposed long before the discovery of the triple-ring nebula (Hillebrandt \& Meyer 1989; Podsiadlowski, Joss \& Rappaport 1990). In this model, the system consisted initially of two massive 
stars, one with a mass of $15-20 M_{\odot}$ and a less massive companion with a mass of $2-5 M_{\odot}$, orbiting with an orbital period of at least 10 years. Because of the relatively long orbital period, these stars would only interact late during the evolution of the more massive star, in fact about 20,000 years before the explosion. When the more massive star starts to transfer mass to the less massive star, mass transfer is unstable, and the more massive star engulfs its less massive companion (see Fig. 7 for a schematic diagram). The less massive star then orbits the core of the more massive star inside the envelope of the more massive component. Because of friction with the envelope, the two stars spiral towards each other and ultimately merge completely, spinning up the envelope in the process and dredging up part of the helium core, explaining the unusual abundances of the supernova (Ivanova \& Podsiadlowski 2003). Because of the dredge-up of the helium and the increased envelope mass, the now single object shrinks to become a blue supergiant. In this transition as much as several solar masses are spun off in the equatorial direction producing a disk-like outflow. In the last 20,000 years before the star explodes, it is a blue supergiant. Such stars have strong stellar winds. This wind sweeps up all the structures that have been produced previously to produce the observed triple-ring nebula (see Morris \& Podsiadlowski [2007] for details).

\section{Type Ia Supernovae}

\subsection{Type Ia Supernovae as Cosmological Distance Candles}

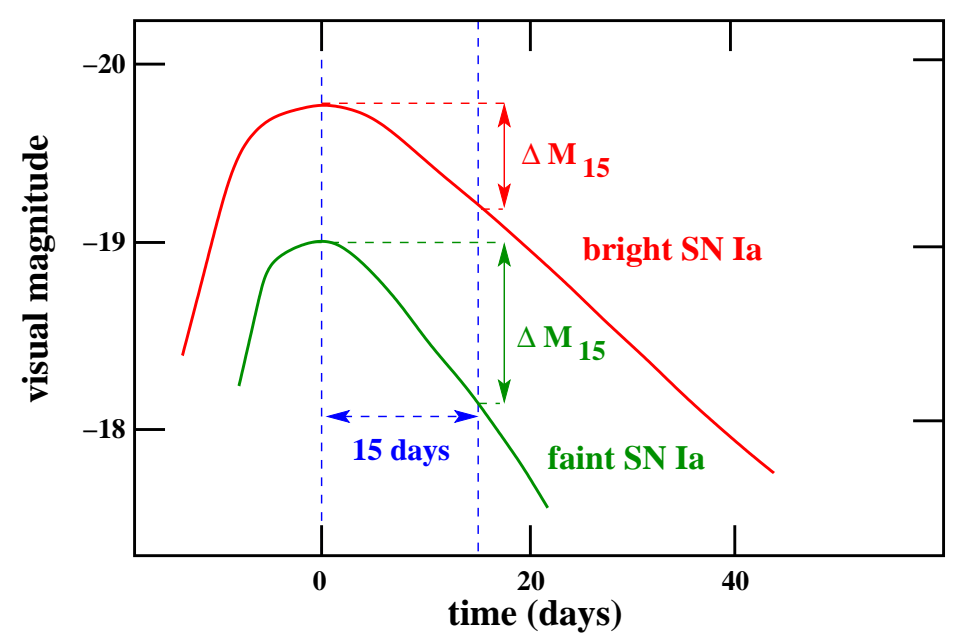

Fig. 8. Visual lightcurves for SNe Ia, illustrating the relationship between peak magnitude and lightcurve width and there use as standardizable cosmological distance candles. 
Type Ia supernovae (SNe Ia) have been very successfully used as standardizable distance candles and have provided the first indication for an accelerating Universe (Riess et al. 1998; Perlmutter et al. 1999). It is sometimes said that SNe Ia are 'standard' candles because they occur in Chandrasekhar-mass white dwarfs with standard properties. This is actually not true; the range of observed peak luminosities is a factor of 10 , reflecting the vastly different amounts of ${ }^{56} \mathrm{Ni}$ produced in different supernovae $\left(0.1-1 M_{\odot}\right)$, a variation that is not at all understood theoretically. However, SN Ia lightcurves vary in a very systematic fashion, where the brighter supernovae have broader lightcurves than their fainter counterparts (as illustrated in Fig. 8). Indeed, empirically there seems to be a one-to-one relationship between the peak magnitude and the lightcurve width which can be measured by the change in magnitude (e.g., in the $B$ band) of the lightcurve between the peak and 15 days after the peak (defining the $\Delta M_{15}$ parameter). This relationship, known as the 'Phillips relation' (Phillips 1993) can be used to determine the true peak magnitude of any SN Ia and makes them useful as cosmological distance candles. The physical origin of the Phillips relation that links the peak SN Ia luminosity to the lightcurve width is now reasonably well understood (see, e.g., Mazzali et al. 2001). The supernova lightcurve is powered by the radioactive decay of ${ }^{56} \mathrm{Ni}$ into ${ }^{56} \mathrm{Co}$ and ${ }^{56} \mathrm{Fe}$, and hence the peak luminosity is directly proportional to the ${ }^{56} \mathrm{Ni}$ mass (Arnett 1982). The lightcurve width on the other hand is determined by the diffusion time, i.e., the timescale on which photons generated by the decay escape from the ejecta. This in turn depends on the opacity, which under these conditions is dominated by iron-group elements (in particular, $\left.{ }^{56} \mathrm{Ni},{ }^{58} \mathrm{Ni},{ }^{54} \mathrm{Fe}\right)$. Since the dominant iron-group element is ${ }^{56} \mathrm{Ni}$, which also determines the lightcurve peak, this introduces a one-to-one relationship between the lightcurve peak and the lightcurve width. However, the opacity is also dependent on other iron-group elements, such as ${ }^{58} \mathrm{Ni}$ and ${ }^{54} \mathrm{Fe}$, which are stable elements and do not provide a radioactive energy source. The ratio of ${ }^{58} \mathrm{Ni}$ to ${ }^{56} \mathrm{Ni}$ depends on the neutron excess $\eta$ in the exploding white dwarf, where $\eta$ can be related to the electron number fraction $Y_{\mathrm{e}}$ by $\eta=1-2 Y_{\mathrm{e}}$. Most importantly, $Y_{\mathrm{e}}$ depends on the initial metallicity of the progenitor and, in particular, on the initial oxygen abundance. Timmes et al. (2003) have estimated the final ${ }^{56} \mathrm{Ni}$ mass fraction as $X\left({ }^{56} \mathrm{Ni}\right) \approx 1-0.054 Z / Z_{\odot}$, where $Z / Z_{\odot}$ is the metallicity relative to solar metallicity. This introduces metallicity as a second, necessary parameter that affects the lightcurve shape. For a reasonable range of metallicity, this may account for the observed spread in the Phillips relation. It is clear now that this metallicity effect needs to be taken into account, in particular, if one wants to improve the use of SNe Ia as cosmological distance candles.

\subsection{The Progenitors of SNe Ia}

One of the weakest points in our understanding of SNe Ia is the nature of their progenitors. There is now broad agreement that most SNe Ia are caused by a 
thermonuclear explosion of a CO white dwarf when its mass approaches the Chandrasekhar mass. What is still uncertain and is indeed controversial is the evolution that produces these Chandrasekhar-mass white dwarfs. The most popular progenitor models fall broadly into two classes, the single-degenerate (SD) model and the double-degenerate (DD) model.

\section{The Single-Degenerate Model}

In the SD model, the white dwarf grows in mass by accreting from a nondegenerate companion star (Whelan \& Iben 1973; Nomoto 1982), where the companion star can either be a main-sequence star, a helium star, a subgiant or even a giant. The main problem with this class of models is that it is generally difficult to increase the mass of a white dwarf by accretion: if the mass-accretion rate is too low, this causes nova explosions and/or helium flashes (Nomoto 1982) which may eject most of the accreted mass. If the massaccretion rate is too high, most of the transferred mass must be lost in a disc wind to avoid a merger of the binary, again leading to a low accretion efficiency. There is only a very narrow parameter range where a white dwarf can accrete hydrogen-rich material and burn in a stable manner. This parameter range may be increased if differential rotation affects the accretion process (Yoon \& Langer 2004). One promising channel that has been identified in recent years relates them to supersoft X-ray sources (van den Heuvel et al. 1992). However, it is not clear whether this channel produces a sufficient number of systems to explain the observed SN Ia rate in our Galaxy $\left(\sim 3 \times 10^{-3} \mathrm{yr}^{-1}\right.$; Cappellaro \& Turatto 1997). On the plus side, a number of binary systems are known that are excellent candidates for SN Ia progenitors: U Sco, RS Oph and TCrB all contain white dwarfs that are already close to the Chandrasekhar mass, where the latter two systems are symbiotic binaries containing a giant companion (see Hachisu et al. 1999 for a discussion of this channel). However, in none of these cases is it clear whether the massive white dwarf is a $\mathrm{CO}$ or an ONeMg white dwarf (the latter is not expected to produce a SN Ia).

\section{The Double-Degenerate Model}

In contrast to the SD model, the DD model (Iben \& Tutukov 1984; Webbink 1984) involves the merger of two CO white dwarfs with a combined mass in excess of the Chandrasekhar mass. This model has the advantage that the theoretically predicted merger rate is quite high, (see, e.g., Yungelson et al. 1994; Han et al. 1995; Nelemans et al. 2001), consistent with the observed SN Ia rate. The main problem with this scenario is that it seems more likely that the disruption of the lighter white dwarf and the accretion of its debris onto the more massive one leads to the transformation of the surviving CO white dwarf into an ONeMg white dwarf which subsequently collapses to form a neutron star (i.e., undergoes accretion-induced collapse) rather than experiencing a thermonuclear explosion (e.g., Nomoto \& Iben 1985), although there may 
be circumstances under which this can be avoided (Yoon, Podsiadlowski \& Rosswog 2007).

In recent years, numerous observational tests have been devised to test these various progenitor models, sometimes with strong claims ("this rules out ..."), but more often with claims that contradict others, and the whole discussion has remained fluid. Some of these tests involve the detetion of circumstellar material, as might be produced by the wind of a single-degenerate donor star, the detection of a surviving companion in a historical supernova remnant in our own galaxy, the direct detection of a progenitor on old plates, and many more.

Two recent SNe Ia have provided important new constraints. The first one was one of the closest SNe Ia in many decades, SN 2011fe/PTF11kly, in M101, the pinwheel galaxy, which reached a peak apparent magnitude of 10 and will remain observable for many years to come. Because M101 is a wellstudied galaxies, there are deep archival HST images of the supernova field. Since these do not show any counterpart at the explosion site, this rules out a single-degenerate progenitor where the donor star is a typical red giant, such as in RS Oph (Li et al. 2011). In contrast, a second recent supernova, PTF $11 \mathrm{kx}$ (Dilday et al. 2012) shows clear evidence for hydrogen in the immediate vicinity of the supernova, which must have been ejected from the progenitor system. The most likely candidate is a red-giant donor with a somewhat larger mass-loss rate than is observed in RS Oph. This clearly demonstrates that there is more than one channel that can produce a normal looking SN Ia.

\section{Gamma-Ray Bursts, Collapsars and Hypernovae}

\subsection{The History of Gamma-Ray Bursts}

Gamma-ray bursts (GRBs) are short flashes of gamma rays, lasting from a fraction of a second to hundreds, sometimes thousands of seconds. They were discovered accidentally in 1967 by a US spy satellite that was scanning the Earth's atmosphere for gamma rays from Russian atmospheric nuclear tests in violation of the atmospheric test-ban treaty. Instead of finding gamma rays from the Russians, the satellite found GRBs from all over the sky. This discovery was kept secret till 1973 (Klebesadel et al. 1973) and remained one of the biggest mysteries in astronomy until 1998. At some point, there were more theories for GRBs than actual bursts. Initially, most people thought that these were probably associated with events on neutron stars in our own galaxy. The mystery only deepended when in the early 90 s a new satellite was launched, NASA's Compton-Ray Observatory, and BATSE, one of its onboard experiments, found that GRBs were not concentrated towards the Galactic disc and centre, as most people had expected, but that their locations were distributed perfectly isotropically all over the sky. This ruled out one of the leading models, neutron stars in the Galactic disc, as sources of these events. It 
still did not fix their distance scale as one can imagine various isotropic distributions. The closest is the Oort cloud of comets around our own solar system, although it is far from clear how these could produce GRBs. A more realistic alternative were sources in the halo of the Milky Way; if the characteristic distance scale of a halo population (e.g., of a special subset of neutron stars or other compact objects) is sufficiently large, it could potentially produce an isotropic sky distribution. The most dramatic possibility was that GRBs originated from the distant Universe and that the GRB isotropy reflected the large-scale isotropy of the Universe. ${ }^{9}$ Depending on the distance scale, GRBs could be associated with feeble events in the outer solar system or with some of the most energetic events in the Universe. One problem with BATSE was that it was not able to localize GRBs very well, at best only to within a few degrees. Such a large field of sky contains far too many sources and makes it impossible to find GRB counterparts in other wavebands.

The resolution of this mystery came with the launch of another high-energy satellite in 1996, BeppoSAX, an Italian-Dutch collaboration, which was able to localize GRBs much better than BATSE (within about 1 arcmin) and, moreover, very quickly, allowing the rapid follow-up with optical telescopes. This quickly lead to the first detection of a GRB in the optical, an optical afterglow associated with a GRB. Moreover, optical spectra of these afterglows showed highly redshifted absorption lines, which must be caused by galaxies in front of the GRBs. This proved beyond doubt that they must originate from cosmological distances, connecting them with the most energetic events in the Universe (apart from the Big Bang itself). Indeed, GRBs are so energetic that they can be detected throughout the observable Universe and can be used as a probe of the early Universe. At present, the most distant explosion in the Universe, and arguably the most distant object known in the Universe, is a GRB at a redshift of 9.4 (Cucchiara et al. 2011).

\subsection{The Main Properties of GRBs}

GRBs come in many different shapes; some show substructure on submillisecond scales, others have a smooth time profile lasting 10 to $100 \mathrm{~s}$ of seconds, perhaps with some short spikes superimposed. Others seem to turn off, only to produce another burst-like event 100s, sometimes 1000s of seconds later. Based on their duration, GRBs are divided into two groups, shortduration GRBs (SGRBs) with an average duration of $0.3 \mathrm{~s}$ and long-duration GRBs (LGRBs) with a median duration of around $20 \mathrm{~s}$, where the spectra of the former group tend to be significantly harder. At present, optical afterglows with intervening absorption lines have only been discovered for LGRBs, though a few SGRBs are found close to big galaxies, making an association

\footnotetext{
${ }^{9}$ Before the advent of BATSE, one of the few astrophysicists who strongly advocated a cosmological origin for GRBs was Bohdan Paczyński whose arguments at the time, however, were not taken very seriously by most people in the field.
} 

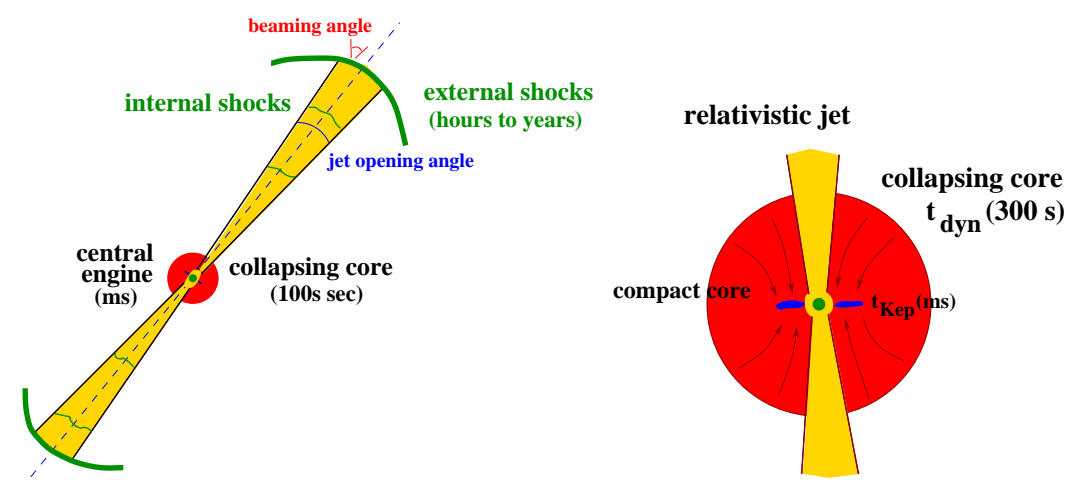

Fig. 9. Cartoon illustrating the origin of long-duration GRBs in the collapsar model.

likely. It is generally believed that the two groups of GRBs are associated with very different events. SGRBs are probably caused by the merger of two compact objects, in particular, two neutron stars or a neutron star and a black hole. In these systems, the two compact objects are driven towards each other by the loss of angular momentum by gravitational radiation, ultimately leading to a cataclysmic merger and a burst of gravitational radiation and gamma rays. In contrast, LGRBs are probably connected with the death of massive stars, as discussed in more detail in $\S 6.4$. To complicate things further, there is another class of GRBs, so-called soft gamma-ray repeaters that have a Galactic origin. They tend to have a softer spectrum than classical GRBs (i.e., SGRBs and LGRBs) and appear to be associated with repeating, non-destructive events occurring on magnetic neutron stars, so-called magnetars. ${ }^{10}$

The spectra of GRBs tend to be quite hard with most of the energy being emitted at $\gtrsim 200 \mathrm{keV}$, and the spectra themselves can best be described by a power law, indicating a mainly non-thermal origin, such as synchroton radiation and/or inverse Compton radiation.

The apparent range of total energy coming out in gamma rays can be large, ranging from $10^{44}$ to several $10^{47} \mathrm{~J}$. However, this assumes that the gamma-ray emission is istropic. In fact, it is generally accepted that GRBs are highly beamed, both relativistically and geometrically.

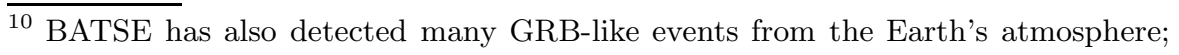
these are associated with thunderstorms in the upper atmosphere. While they cannot be mistaken for classical GRBs because of their location, it is amusing that Nature produces events that look very similar in terms of durations and observable gamma-ray fluxes, but have very different underlying mechanisms on hugely varying energy scales.
} 


\section{Relativistic Beaming of GRBs}

Detailed models of GRBs indicate that the material that emits the gamma rays moves relativistically with Lorentz factors $\gamma \gtrsim 100$. This implies that the radiation is relativistically beamed with most of the emission confined to a narrow beam in the direction of the flow with a beaming angle $\theta_{\text {rel }} \lesssim 1 / \gamma$. Since the flow itself is believed to be a confined to a narrow jet with an opening angle $\theta_{\text {jet }} \sim 1-10^{\circ}$, only an observer within the beam will be able to observe a GRB (see Fig. 9). This has two important implications: (1) since the energy is not isotropic, the true energy in a GRB will be reduced by a factor of $4 \pi / \Delta \Omega$ over the naive estimate based on isotropic emission, where $\Delta \Omega$ is the effective solid angle of the beam. (2) Since the beaming angle is small, most GRBs are not observable (at least not in gamma rays). This implies that the true GRB event rate has to be increased by the same factor $4 \pi / \Delta \Omega$. Initially, $\theta_{\text {rel }}$ will generally be less than $\theta_{\text {jet }}$. However, as the material in the jet is slowed down, most likely because of an interaction with the circumstellar medium, there will come a point when $\theta_{\text {rel }}$ becomes larger than $\theta_{\text {jet }}$, which means that the afterglow emission is spread over a larger solid angle. This produces a characterstic break in the afterglow lightcurve and can be used, in combination with detailed modeling of the afterglow, to estimate the jetopening angle and hence the beaming correction (typically a factor of a few 100). Indeed, when this is done, this reduces the estimate of GRB energies to a much narrower range around $10^{44} \mathrm{~J}$ (Frail et al. 2001), similar to the energy in a normal supernova. ${ }^{11}$ However, this accounts only for the energy in gamma rays; the total energy in the GRB event, including the energy in the jet itself and any possibly associated supernova event, is probably at least an order of magnitude larger (see $\S 6.5$ ). It also means that, while there are about 3 GRBs in the Universe per day that we can detect in principle, the true rate is at least a few 100 times larger. This still makes GRBs extremely rare events; for comparison, core-collapse supernovae are about a factor of 1000 more common. Therefore, whatever produces a GRB clearly requires very special circumstances.

\subsection{The Relativistic Fireball Model}

\section{The Compactness and the Baryon Loading Problem}

GRBs are variable on timescales of $t_{\text {min }} \sim 10^{-3}$ s. This implies that the emitting region, which should scale like $c t_{\text {min }}$ (where $c$ is the speed of light) is $\sim 10^{5} \mathrm{~m}$. However, this causes an immediate problem; if one confined all the photons emitted in a GRB to a sphere of that radius, this photon gas would be

${ }^{11}$ NASA's Swift satellite, launched in 2004, has only been able to detect clear jet breaks in a few GRBs, despite its much better sensitivity. It is presently not clear what implications this has for estimates of the jet opening angle and the beaming correction that needs to be applied. 
optically thick to the production of electron/positron pairs (since most photons have energies larger than $0.5 \mathrm{MeV}$ ). This has several consequences. (1) The typical photon energy is rapidly downgraded to energies $<0.5 \mathrm{MeV}$. (2) The gamma-ray energy is converted into kinetic energy (of the $\mathrm{e}^{+} \mathrm{e}^{-}$plasma). (3) The resulting spectrum becomes that of a modified blackbody, which is not what is observed.

In addition, this emitting region has to be essentially baryon-free. Otherwise, the gamma-ray energy would mainly go into accelerating the baryons. What is needed is an essentially baryon-free relativistic fireball consisting only of electrons, positrons and gamma-ray photons.

Having such a fireball confined to a jet alleviates the problem slightly as it reduces the rate of pair production, but does not solve it.

\section{The Role of Shocks}

The solution of this conundrum is that indeed the energy that is produced in the central GRB engine goes first into the kinetic energy of the relativistic jet, but that this energy is later reconverted into photons by shocks after the flow has become optically thin (Meszaros \& Rees 1993), e.g., emitted in the form of synchroton radiation in the shocked material. Two types of shocks may play a role in this picture: external shocks caused by the fireball running into an external medium and internal shocks in the relativistic flow itself, as faster portions of the flow catch up with slower ones (see Fig. 9). It is easy to imagine that a combination of the two types of shocks can account for the large diversity of observed GRB profiles, where the internal shocks may account for a lot of the fine structure of the bursts, while the external shocks are more likely to produce the broader structures. Even though this fireball model does not depend on the details of the central engine that actually drives the GRB, the engine still has to be variable on very short timescales to produce the observed variations in the flow and its interaction with the surrounding medium.

\subsection{The Collapsar Model and the Central Engine}

Observationally, long-duration GRBs involve processes that occur on timescales that vary by many orders of magnitude (see Fig. 9). The central engine that drives the GRB operates on a characteristic timescales of milliseconds with sub-millisecond variations, while the main GRB must last for 10 to $10^{3} \mathrm{~s}$. The longest timescale associated with the optical afterglow (hours to years) is probably the easiest to understand; in the framework of the relativistic fireball model, it is just determined by the interaction of the relativistic jet with the surrounding medium ( $\S 6.3$ ) and any supernova event possibly associated with the GRB ( $\S 6.5)$. The presently favoured model for the GRB itself is the collapsar model (Woosley 1993), which connects a long-duration GRB with 
the collapse of the rapidly rotating core of a massive star to a black hole. ${ }^{12}$ Because of its rapid rotation, such a core cannot collapse directly to a black hole since angular momentum has to be conserved in a dynamical collapse. While the low-angular-momentum material along the polar region can collapse directly to form a proto-neutron star or black hole at the centre, most of the high-angular-momentum material first has to first form a centrifugally supported disc orbiting the central object. Viscous processes within the disc will then allow accretion of this material onto the black hole. The characteristic timescale associated with this is the Keplerian timescale near the central compact object which for a neutron star or low-mass black hole is of order milliseconds. On the the hand, the overall duration of the accretion activity is determined by the collapse timescale of the core which is of order its dynamical timescale, which can be estimated as $t_{\mathrm{dyn}} \sim 1 / \sqrt{4 G \rho}$, where $\rho$ is the mean density of the core. Taking the typical density of a collapsing helium star as $\rho_{\mathrm{c}} \simeq 4 \times 10^{4} \mathrm{~kg} \mathrm{~m}^{-3}$, then yields a characteristic collapse timescale of $t_{\mathrm{dyn}} \sim 300 \mathrm{~s}$, very much consistent with the characteristic observed duration of LGRBs. Thus, the collapsar model naturally explains both the overall duration of an LGRB as well as its short-term variability. Note that the accretion rate onto the central object is generally determined by the viscous processes in the disc and not the collapse of the core itself. If the central accretion rate is lower than the overall infall rate, as is particularly likely to happen in the early phase of collapse, the mass in the disc will increase until the disc itself becomes gravitationally unstable (via the Toomre instability; Toomre 1964). The disc then becomes globally unstable and will develop spiral modes that very efficiently redistribute angular momentum in the disc (on a dynamical timescale and not the natural viscous timescale of the disc). This leads to a sudden increase in the central accretion rate $\left(\gtrsim 1 M_{\odot} / \mathrm{s}^{-1}\right)$, reflected in a sharp increase in GRB activity (for details see Taylor et al. 2011). In addition, thermal instabilities in such an unstable disc cause clumping of disc material and, as these clumps are accreted, could cause variability on sub-millisecond timescales.

A necessary requirement for the collapsar model is that the collapsing core has enough angular momentum to be able to form a disc. We can estimate the amount required by considering the case of forming a disc around a black hole of mass $M_{\mathrm{BH}}$ that formed in the initial collapse. Treating this in a completely Newtonion manner, the specific angular momentum of a Keplerian disc is given by $j=\sqrt{G M_{\mathrm{BH}} R_{\text {in }}}$, where $R_{\text {in }}$ is the inner radius of the disc. Taking this radius to be the last stable orbit around a Schwarzschild black hole,

${ }^{12}$ The collapsar model is not the only model presently under consideration. One promising alternative involves the formation of a rapidly rotating neutron star with a very strong magnetic field, a magnetar, which is spun down on a timescale of a few seconds, extracting a large fraction of the rotational energy and powering a GRB (see, e.g., Metzger et al. 2011). Similar to the collapsar model, it requires a rapidly rotating progenitor core, but the final object is more likely to be a neutron star. 
$R_{\text {in }}=6 G M_{\mathrm{BH}} / c^{2}$, the critical $j$ for disc formation can be written as $j_{\text {crit }}=$ $\sqrt{6} G M_{\mathrm{BH}} / c \simeq 2 \times 10^{12} \mathrm{~m}^{2} \mathrm{~s}^{-1}\left(M_{\mathrm{BH}} / 2 M_{\odot}\right)$. This estimate gives the specific angular momentum required in the outer parts of the progenitor's core.

\section{The GRB Energy Source}

In order to produce an energetic GRB, more energy is required than in an ordinary supernova (taking into account that only a fraction of the total energy emerges in gamma rays). There are two potential energy sources. The first is the gravitational binding energy of the disc that is released as material is accreted onto the central black hole. In terms of the rest-mass energy of the accreted material, $M_{\text {acc }}$, this can be written as

$$
E=\eta M_{\mathrm{acc}} c^{2} .
$$

For a non-rotating Schwarzschild black hole, the efficiency factor $\eta \simeq 0.06$, as determined by the binding energy at the last stable orbit. For a rotating Kerr black hole, $\eta$ can, in principle, be as high as $42 \%$, although in practice it is unlikely to be larger than $\sim 30 \%$. For a disc mass of $\sim 1 M_{\odot}$, this means that more than $10^{46} \mathrm{~J}$ could potentially be extracted from the disc.

The second source of energy is the spin energy of the black hole itself. If the material that is accreted by a rotating black hole is magnetically connected to the disc surrounding the black hole (provided the disc has a strong poloidal magnetic field), this causes a magnetic coupling of the black hole's ergosphere with the disc, which allows black-hole spin energy to be extracted. This is known as the Blandford Znajek mechanism (Blandford \& Znajek 1977). Up to $29 \%$ of the spin energy can in principle be extracted, where more generally the extractible energy is given by

$$
E_{\mathrm{BZ}}=5 \times 10^{46} \mathrm{~J} f(a)\left(M_{\mathrm{BH}} / M_{\odot}\right),
$$

where $f(a)=1-\left(\left[a+\sqrt{1-a^{2}}\right] / 2\right)^{1 / 2} \leq 0.29$, and $a$ is the angular momentum parameter that can vary between 0 and 1 .

For both energy sources, there is more than enough energy to power even a very powerful GRB. The key is that, because of the rotation, a much larger fraction of the gravitational binding energy of the compact object being formed can be used than in a classical neutrino-driven explosion, where most of this energy just escapes in the form of neutrinos and is wasted.

\section{The Production of a Relativistic Jet}

Relativistic jets are a generic feature of most GRB models; how they are produced is less clear. One possibility is that they are neutrino-driven jets. Just, as in the case of a normal core-collapse supernova, neutrinos are copiously produced and in fact provide the main cooling process. Most of these neutrinos will escape from the system, but those that interact with other neutrinos 
will do so preferentially along the polar axis (for geometrical reasons) producing $\mathrm{e}^{+} / \mathrm{e}^{-}$pairs. Since this is also the region with low baryon loading, this could potentially drive a relativistic jet. It is not clear whether this process is efficient enough in the case of LGRBs, but could be in the case of merging neutron stars in the context of SGRBs. For LGRBs it appears more likely that the relativistic jets are produced by some, presently not well understood MHD process as matter is accreted at a very high rate.

\subsection{Hypernovae: the LGRB - Supernova Connection}

In the late 90s it was realised that, in addition to the normal core-collapse and thermonuclear explosions, there are more energetic supernovae with an energy output $\gtrsim 10^{45} \mathrm{~J}$, i.e., they are at least 10 times as energetic as a normal supernova. These are now often referred to as hypernovae (HNe) or alternatively as broad-lined supernovae, since they have very broad lines, reflecting their more energetic nature. The proto-type is SN 1998bw (Iwamoto et al. 1998). SN 1998bw was also observed as an LGRB, establishing the first connection between a GRB and a supernova, the death of a massive star. Admittedly, the GRB associated with SN 1998bw was rather feeble, causing some arguments in the literature about the validity of this link, but there have been many more GRB/SN connections since, in some cases involving very typical GRBs (e.g. SN 2009nz/GRB 091127; Berger et al. 2011). This leaves no doubt that at least some LGRBs are associated with the explosions of massive stars. In addition, many GRB afterglows show bumps in the lightcurve that are consistent with an underlying hypernova-like event. Interestingly, at present all GRB supernovae are classificed as SNe Ic, i.e., supernovae that have lost both their hydrogen and their helium envelopes, providing a potentially important clue to the nature of their progenitors. Hypernovae are just as rare as LGRBs with a characteristic rate for a galaxy like the Milky Way of $10^{-5} \mathrm{yr}^{-1}$ (Podsiadlowski et al. 2004). This, however, does not mean that all LGRBs are accompanied by a hypernova. In order to see a hypernova, the event has to produce a large amount of ${ }^{56} \mathrm{Ni}$, since it is the radioactive decay of ${ }^{56} \mathrm{Ni}$ that powers a SN Ic lightcurve. However, Ni production is not required in the collapsar model; indeed Woosley (1993) originally suggested that this model would produce a faint supernova or no supernova at all. At present, it is not clear where $\mathrm{Ni}$ is produced in the collapsar model, and it is conceivable that in some cases no or very little $\mathrm{Ni}$ is ejected, leading to a dark GRB burst, i.e., a GRB not accompanied by a bright afterglow or hypernova.

One would also not necessarily expect every hypernova to be associated with a GRB, even if the hypernova is created by a central engine. In order to observe a GRB, a relativistic jet has not only to form but also has to escape from the collapsing star, i.e., be able to penetrate the remaining envelope. If the jet fails to escape, it may still drive a powerful explosion, but may not be accompanied by a GRB: it will be a failed GRB. 
Even though hypernovae are rare today, if they were more common in the past (e.g., because a lower metallicity makes them more common), they could be important contributors to the global nucleosynthesis as they produce a lot of $\mathrm{Fe}$ (from the decay of $\mathrm{Ni}$ ) at very early times and other elements like $\mathrm{Zn}$, that are overproduced in such energetic explosions.

\subsection{The Progenitors of GRBs}

For the case of short-duration GRBs, there is broad agreement that they are probably associated with the merger of two compact objects, two neutron stars, a neutron star and a black hole, or perhaps a neutron star and a white dwarf. The situation is very different for long-duration GRBs, where there is not even agreement whether they involve single stars or binaries. In either case, as GRBs are rare, there have to be special circumstances that produce a GRB progenitor.

\section{Single-Star Progenitor Models for LGRBs}

The problem with single stars is that they very efficiently lose their angular momentum in stellar winds. Moreover, even very weak seed magnetic fields efficiently redistribute angular momentum in a star (trying to erase any differential rotation), which prevents the formation of a rapidly rotating core (see Heger et al. 2005). This problem is less severe at lower metallicity where the wind mass-loss rates are much lower. Moreover, if stars rotate extremely rapidly, they may evolve homogeneously and avoid a red-supergiant phase altogether (see $\S 7.3$ ) and the associated strong mass loss. Yoon \& Langer (2005b) and Woosley \& Heger (2006) have proposed that such homogeneous evolution can produce rapidly rotating progenitor cores, provided that the metallicity is quite low, typically less than $1 / 5$ solar (Yoon et al. 2006). Indeed, there has been some observational evidence that LGRBs are preferentially found in small galaxies with low metallicity, although in recent years it has become clear that LGRBs can also occur at solar or even super-solar metallicity (e.g., Levesque et al. 2010). Therefore it seems from observations that there is no strong metallicity cutoff for GRBs, but perhaps just a metallicity bias.

\section{Binary Progenitor Models}

It some sense, it seems much easier to produce a GRBs in a binary as there always is an overabundance of stored angular momentum in the form of the orbital angular momentum of the binary: one just has to be able to tap that orbital angular momentum at the right time to produce a rapidly rotating stellar core (within the framework of the collapsar model). But even this is not so easy, as the stars in binaries are subject to the same processes that spin down single stars, such as stellar winds and magnetic coupling. 
Considering the complexities of binary evolution it is perhaps not surprising that there is large number of proposed binary scenarios which we can only sketch here briefly (for a detailed review see Fryer et al. 2007).

In many respects the simplest binary process that can produce a rapidly rotating helium star is tidal spin up since, in a tidally locked binary, a star can be spun up (or down) until its spin angular velocity is equal to the orbital angular velocity (e.g., Izzard et al. 2004; Detmers et al. 2008). Simple angularmomentum estimates suggest that this requires an orbital period shorter than $\sim 10 \mathrm{hr}$. In practise, this means that the companion is most likely a compact object (a neutron star or a black hole), similar to the X-ray binary Cygnus X3. However, Detmers et al. (2008) found that, at solar metallicity, the expected strong wind from the Wolf-Rayet star leads to a significant widening of the binary and the ultimate spin-down of the companion. As a consequence, this channel is only likely to work at low metallicity.

Most binary models for LGRBs proposed to date involve the merger of two stars of some type, e.g., a compact object (neutron star or black hole) with a helium core, two helium cores, a helium core and a CO core (see Fryer et al. 2007 for detailed discussions and references). This is a particularly efficient way for converting orbital angular momentum into spin angular momentum. These models work best if the interaction occurs late in the evolution of the progenitor (so-called Case C mass transfer; see $\S 7.1$ ) as there is little remaining time after the merger to spin down the merger product in a strong Wolf-Rayet wind.

Observationally all GRB-related supernovae to date have been identified as SNe Ic, i.e., involve progenitors that have lost both their hydrogen and their helium envelopes, an issue that it is not usually addressed in these models. There has been some debate of how much helium could be hidden in a SN Ic. The most recent estimates suggest that it could be only very little (less than a few $0.1 M_{\odot}$; Hachinger et al. 2012); this provides a serious challenge to all the models mentioned so far, single and binary.

This problem could potentially be avoided if the merger itself is explosive. Podsiadlowski et al. (2010) proposed an explosive common-envelope ejection scenario in certain types of late binary mergers, where the merging of a hydrogen-rich low-mass star with an evolved massive helium core triggers a thermonuclear runaway in the helium layer, leading to the ejection of both the hydrogen envelope and the helium layer. As the CO core is moderately spun up in the process, this could provide a viable (though probably not very common) channel for a GRB associated with a SN Ic.

\section{The Diversity of Supernova Explosions}

\subsection{The Role of Binarity}

While it has been clear for many years that binary interactions strongly affect the structures of stellar envelopes, both by mass loss and by mass accretion, 
and hence are likely to be a major cause for the observed diversity of supernova sub-types, it has only recently become clear that they can also alter the core evolution and, in fact, the final fate of a star. Generically, one expects that, if mass loss/accretion occurs during an early evolutionary phase, the core continues to evolve subsequently like a less or more massive star. However, this is not true if mass loss occurs after the main-sequence phase.

\section{Types of Binary Interactions}

Classification of Roche-lobe overflow phases

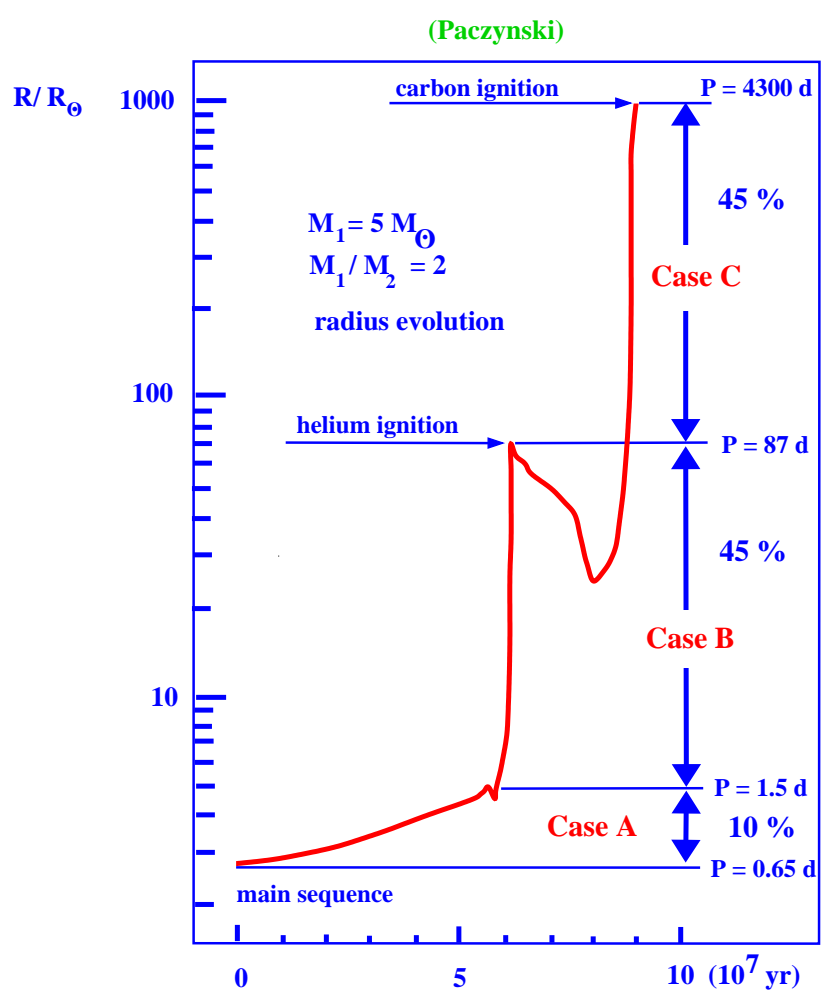

Fig. 10. The evolution of the radius of a $5 M_{\odot}$ star as a function of its lifetime to illustrate the ranges in radius and orbital period for the different cases of RLOF phases, as indicated, assuming a $2 M_{\odot}$ companion.

While most stars in the sky are probably in binary system, the only ones we are interested here are those where at least one of the components transfers mass to the other one by RLOF. The fraction of massive binaries that interact at some point during their evolution has been estimated to be in the range 
of $30-50 \%$ of all stellar systems, where the more massive stars are likely to interact more frequently. Indeed, there is even some evidence that for binaries containing $\mathrm{O}$ stars the fraction of interacting binaries may be as high as $75 \%$. For the first phase ${ }^{13}$ of mass transfer for one of the stars, one distinguishes three cases of mass transfer depending on the nuclear evolutionary state of the star: Case $A$ (the star is on the main sequence burning hydrogen), Case $B$ (the star has finished hydrogen burning, but not helium burning in the core), Case $C$ (the star has completed core helium burning). Fig. 10 shows the radius evolution of a $5 M_{\odot}$ star as a function of time and indicates the range where the different cases occur. Since the radius of the star expands only very little (a factor of $\sim 2$ ) on the main sequence but a factor of more than 10 before helium ignition and again after helium burning, it is much more likely that RLOF starts after the star has completed its main-sequence phase (this assumes a logarithmically flat initial period distribution). On the other hand, since a star spends most of its life on the main sequence, it follows that most binaries observed in the sky have not yet had a strong binary interaction, but many of them will do so in the future. This is particularly important when studying the end states of stars and supernovae that probe the late evolutionary phases of a star. Note also that quite massive stars $\left(\gtrsim 20 M_{\odot}\right)$ tend to expand only moderately after helium core burning, and hence, for massive stars, Case C mass transfer tends to be much less important than Case B mass transfer, where most of the expansion occurs.

When RLOF occurs, one has to distinguish between different modes of mass transfer, depending on whether mass transfer is stable or unstable with very different outcomes.

Stable mass transfer

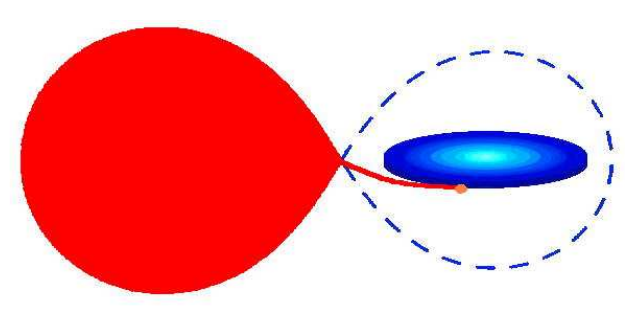

Fig. 11. Cartoon illustrating stable mass transfer.

Stable, (quasi-)conservative mass transfer (as illustrated in Fig. 11) is the easiest type of mass transfer to understand. In this case, most, but not necessarily all, of the transferred mass is accreted by the companion star, generally leading to a widening of the binary. Mass transfer ends when most of the

\footnotetext{
${ }^{13}$ If a star experiences more than one mass-transfer phase, the nomenclature quickly becomes complicated, and there is no established standard notation.
} 


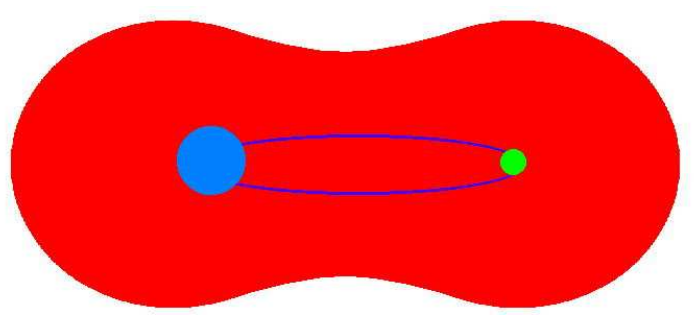

Fig. 12. Cartoon illustrating unstable mass transfer.

hydrogen-rich envelope of the donor star has either been transferred to the companion or been lost from the system. The end product will be a hydrogenexhausted helium star with at most a small hydrogen-rich envelope. ${ }^{14}$ Mass accretion will also change the structure of the accreting star. If it is still on the main sequence, the accretor tends to be rejuvenated and then behave like a more massive normal main-sequence star. On the other hand, if it has already left the main sequence, its evolution can be drastically altered, and the star may never evolve to become a red supergiant, but explode as a blue supergiant (if it is a massive star; Podsiadlowski \& Joss 1989).

This is illustrated in Fig. 13 which shows the evolutionary tracks of both components in a binary in a model that reproduces the properties of the progenitor system of SN 1993J, a Type IIb supernova in M81 (Maund et al. 2004). The primary with an initial mass of $15 M_{\odot}$ starts to experience stable mass transfer near the end of its evolution (late Case $\mathrm{C}$ mass transfer), but still has a small envelope of $0.3 M_{\odot}$ left at the time of the explosion, consistent with the requirement for a SN IIb (see $\S 3.3$ ). The companion accretes a large fraction of this mass, but because its initial mass $\left(14 M_{\odot}\right)$ is close to the mass of the primary (in order to ensure stable mass transfer), it has already left the main sequence and because of the accretion never becomes a red supergiant. It will spend the rest of its evolution as a blue supergiant and finally explode in a supernova similar to SN 1987A ( $§ 4)$. Thus, this single observed system illustrates two ways in which binary evolution produces a particular supernova sub-type.

\section{Unstable mass transfer and common-envelope evolution}

Mass transfer is unstable when the accreting star cannot accrete all off the material transferred from the donor star. The transferred material than piles up on the accretor and starts to expand, ultimately filling and overfilling the accretor's Roche lobe. This leads to the formation of a common-envelope (CE) system, where the core of the donor and the companion form a binary

${ }^{14}$ Stable mass transfer can also occur for an expanding hydrogen-exhausted helium star (so-called Case BB mass transfer). In this case, the star is likely to lose a large fraction/most of its helium envelope. This can produce a SN Ic progenitor with very low ejeta mass. 


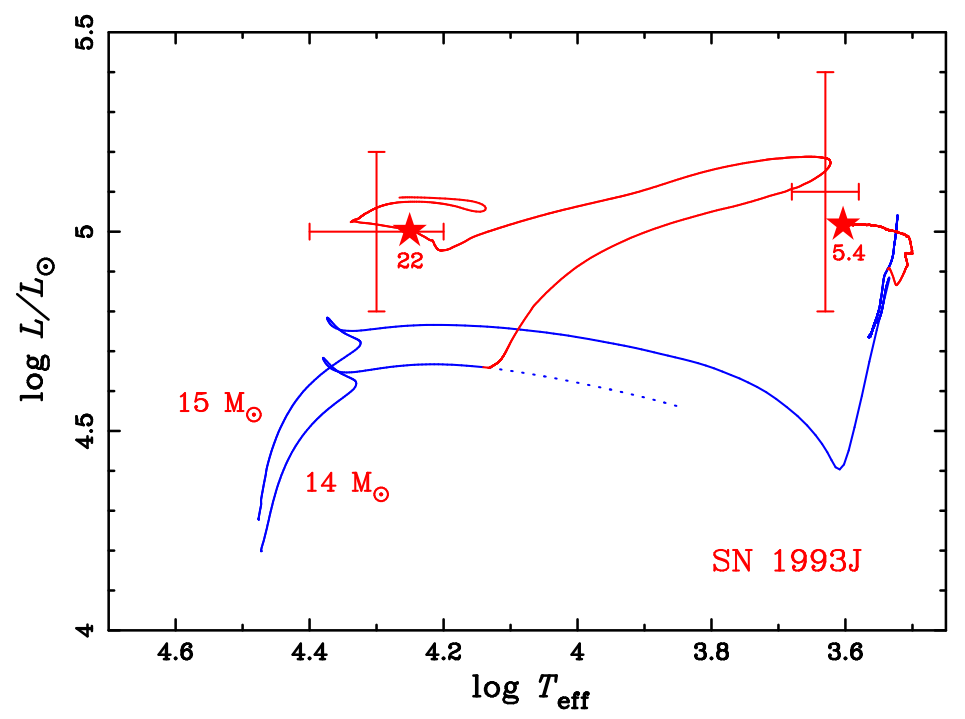

Fig. 13. Hertzsprung-Russell diagram showing the evolution of the two components of a massive binary that experiences stable Case $\mathrm{C}$ mass transfer. At the time of the explosion, the primary is a $5.4 M_{\odot}$ star with a small $0.3 M_{\odot} \mathrm{H}$-rich envelope. Because of the accreted mass the secondary never becomes a red supergiant and explodes as a blue supergiant similar to SN 1987A. The locations of the two components in the observed progenitor system of the IIb supernova SN 1993J are indicated by large error bars. (From Maund et al. 2004.)

immersed in the envelope of the donor star (see Fig. 12). Friction between the immersed binary and the envelope will make the two components spiral towards each other until enough orbital energy has been released to eject the envelope (Paczyński 1976). This ends the spiral-in phase and leaves a much closer binary consisting of the core of the giant and a normal-star secondary. If the core is massive enough to explode in a supernova, it will produce a $\mathrm{SN}$ Ib or Ic. Since this spiral-in phase is very short-lived, the immersed companion star will not be able to accrete much matter and will emerge little changed from the CE phase.

\section{Binary mergers}

The most dramatic consequence of a CE phase is that the orbital energy that is released in the spiral-in phase in not sufficient to eject the envelope. In this case, the spiral-in process continues till the core of the donor has merged with the companion, producing a single, initially rapidly rotating star (such as FK Comae stars).

Binary mergers are one of the least studied phases of binary evolution. Despite of their lack of attention, binary mergers are by no means rare events: estimates based on binary population synthesis (BPS) studies suggest that $\sim$ $5-10 \%$ of all stars experience a complete merger with a companion star during 
their evolution and this fraction could be substantially higher for binaries containing $\mathrm{O}$ stars.

\section{Black Hole or Neutron Star?}

Binary interactions can also drastically change the core evolution of a massive star, altering its final fate. This is particularly true if a star loses its hydrogen envelope just before or early during He-core burning (early Case B mass transfer).

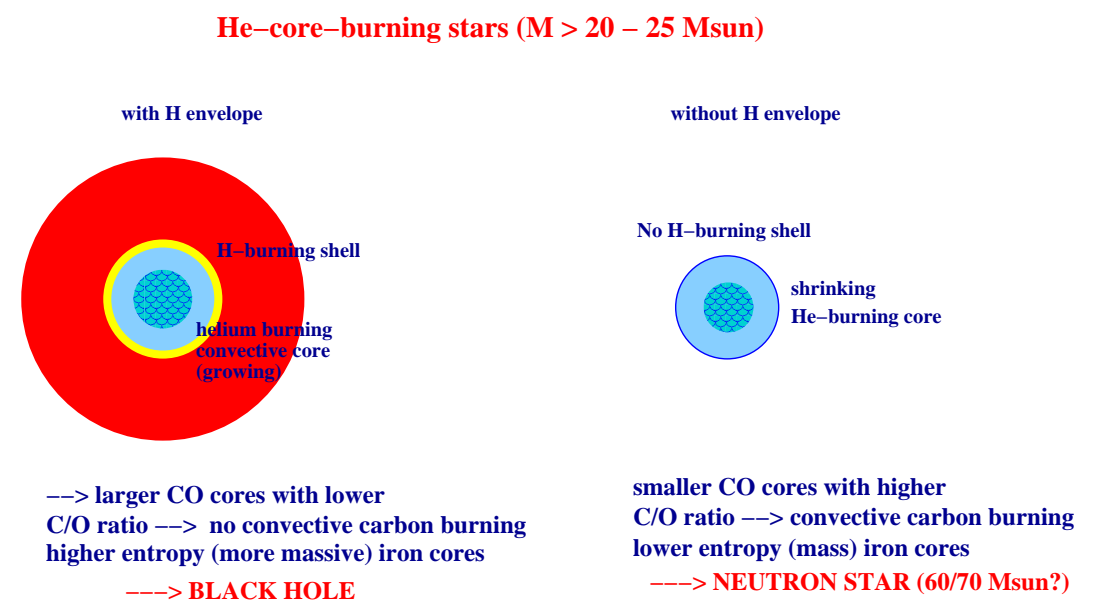

Fig. 14. Schematic comparison of the helium core-burning phase of stars with (left) and without a hydrogen envelope (right) for massive stars $\left(M \gtrsim 20 M_{\odot}\right)$. Because of the lack of a H-burning shell, stars without a hydrogen envelope produce smaller He-exhausted cores with a larger $\mathrm{C} / \mathrm{O}$ fraction. This makes them more likely to ultimately collapse to a neutron star instead of a black hole.

First, consider the case of a massive star $\left(>20-25 M_{\odot}\right)$ that, if single, would be likely to eventually collapse to a black hole (Fryer \& Kalogera 2001). However, if it loses its H-rich envelope early enough, its evolution is quite different (see Fig. 14). Because of the lack of a H-burning shell, the convective core does not grow during helium core burning, and stars end up with much smaller CO and ultimately iron cores (Brown et al. 2001). Indeed, because of this, such $\mathrm{H}$-deficient stars formed in Case B binaries are expected to end their 
evolution as neutron stars rather than as black holes (Brown et al. 2001), even for initial masses as high as $\sim 60 M_{\odot}$. In contrast, single stars are believed to produce black holes for much lower initial masses $\left(\sim 20-25 M_{\odot}\right.$; e.g., Fryer \& Kalogera 2001). On the other hand, single stars only become Wolf-Rayet stars if their initial mass is larger than $\sim 25-35 M_{\odot}$ (depending on the exact massloss rate). Since the formation of a slowly rotating black hole is not apriori expected to be associated with a bright supernova (as the whole star can just collapse into a black hole), this has the important implication that all normal $\mathrm{H}$-deficient core-collapse supernovae (SNe Ib/Ic) may require a close binary companion.

\section{Electron-Capture Supernovae in Close Binaries}

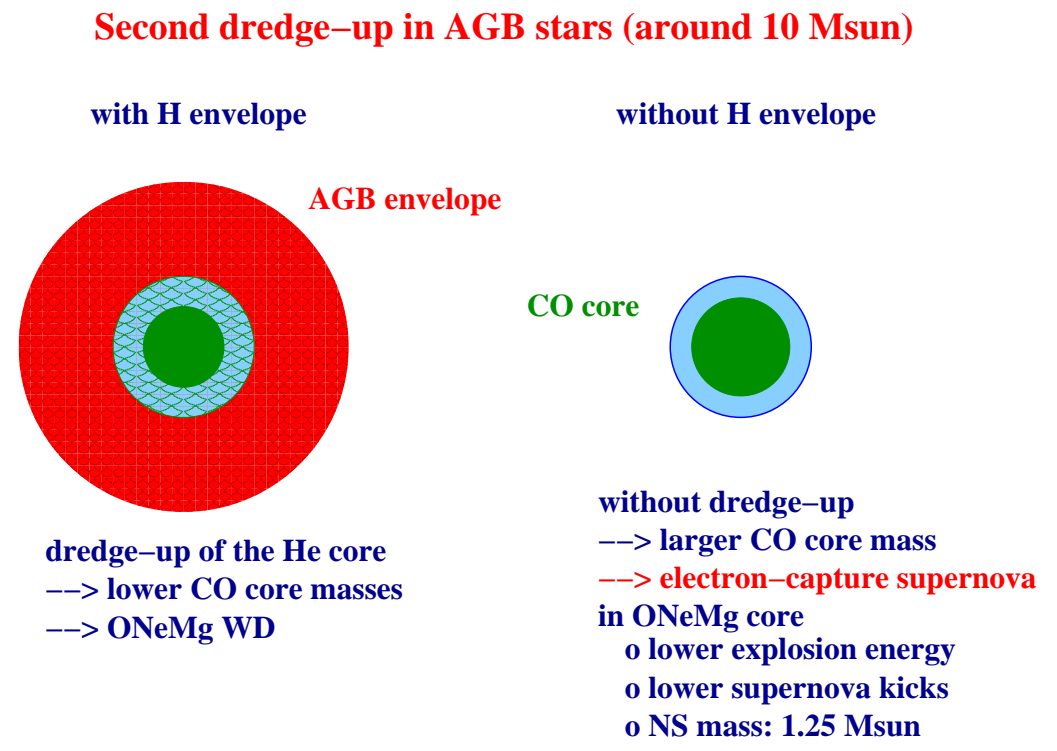

Fig. 15. Schematic comparison of the late evolution of stars with an initial mass around $10 M_{\odot}$ with and without a H-rich envelope. The former experience a so-called second dredge-up phase at the beginning of the AGB phase reducing the size of the helium core; these stars are therefore more likely to end up as $\mathrm{ONeMg}$ white dwarfs. On the other hand, stars without the second dredge-up may eventually collapse and experience an electron-capture supernova.

Another mass range where binary interactions can drastically change the final fate of a massive star is near the minimum mass for stars to explode as supernovae (around $7 M_{\odot}$, where the exact value depends on the amount 
of convective overshooting and the metallicity of the star). Single stars in this mass range experience a second dredge-up phase when they ascend the asymptotic giant branch (AGB), where a large fraction of the $\mathrm{H}$-exhausted core is dredged up and mixed with the envelope. This reduces the core mass at the end of the AGB phase; as a consequence, single stars as massive as $10 / 11 M_{\odot}$ probably produce $\mathrm{ONeMg}$ white dwarfs rather than a supernova. In contrast, if such stars lose their H-rich envelopes due to a binary interaction before reaching the AGB, they end up with much larger He cores and are likely to produce an electron-capture (e-capture) supernova (see $\S 7.2$ for further discussion).

To summarize the effects of binary interactions on the final fate of stars, Table 2 contrasts the expected differences for stars in single systems/wide binaries (which includes Case C systems) to stars in close binaries.

\begin{tabular}{|c|c|c|}
\hline & single/wide binary & close binary \\
\hline CO white dwarf & $<7 M_{\odot}$ & $<7-17 M_{\odot}$ \\
\hline ONeMg white dwarf & $7-10 M_{\odot}$ & $7-8 M_{\odot}$ \\
\hline Neutron star: & & \\
\hline electron-capture & $\sim 10 M_{\odot}$ & $7 / 8-10 M_{\odot}$ \\
\hline iron core collapse & $10-20 / 25 M_{\odot}$ & $10-60 M_{\odot}$ \\
\hline Black hole: & \multirow{3}{*}{\multicolumn{2}{|c|}{$\begin{array}{l}20 / 25-40(?) M_{\odot} \gtrsim 60 M_{\odot}(?) \\
\quad \gtrsim 40 M_{\odot}(?)\end{array}$}} \\
\hline two-step & & \\
\hline prompt & & \\
\hline no remnant (Z?) & \multicolumn{2}{|c|}{$\gtrsim 140 M_{\odot}$} \\
\hline
\end{tabular}

Table 2. Final Fate of Stars: Single vs. Binary.

\subsection{The Diversity of Supernova Explosions}

As has already become clear from the discussion so far, there is more to supernovae than just two standard explosion types. This section summarizes all the main explosion types presently being considered.

\section{Neutron Stars}

Iron core collapse

The collapse of a massive iron core to a neutron star, leading to a neutrinodriven explosion (see $\S 2.1$ ) is the expected standard fate for the majority of massive stars, both single and binary. The typical supernova energy of these explosions is $\sim 10^{44} \mathrm{~J}$, as set by the binding energy of the inner part of the ejecta.

Electron-capture (e-capture) supernovae 


\begin{tabular}{llcl}
\hline Neutron Star & $\begin{array}{l}\text { Fe core collapse } \\
\text { electron capture }\end{array}$ & $\begin{array}{c}10^{44} \mathrm{~J} \\
\vdots 10^{43} \mathrm{~J}\end{array}$ & $\begin{array}{l}\text { large SN kick } \\
\text { small SN kick }\end{array}$ \\
& magnetar & no SN & \\
\hline Black Hole & prompt & faint SN & \\
& fallback & $\gtrsim 10^{44} \mathrm{~J}$ & GRB related \\
& collapsar & & \\
& (engine-driven) & $10^{44} \mathrm{~J}$ & no remnant \\
thermonuclear & runaway C burning & $\lesssim 10^{43} \mathrm{~J}$ & WD remnant (?) \\
& He detonation on WD & $\gg 10^{44} \mathrm{~J}$ & no remnant \\
\hline pair-instability & runaway O burning & $\gg 1$
\end{tabular}

Table 3. Summary of Explosion Types.

A neutron star can also form by the collapse of a very degenerate ONeMg core, long before an iron core has developed. This is the expected fate for stars around $\sim 10 M_{\odot}$. In this case, the collapse is triggered by the sudden capture of electrons onto $\mathrm{Ne}$ and $\mathrm{Mg}$ nuclei taking away the hydrostatic support provided by the degenerate electrons (Nomoto 1984). This occurs at a characteristic density $\left(\sim 4.5 \times 10^{12} \mathrm{~kg} \mathrm{~m}^{-3}\right.$; Podsiadlowski et al. 2005), which can be related to a critical pre-collapse mass for the ONeMg core of $\sim 1.37 M_{\odot}$. Since, in this case, most of the core collapses to form a low-mass neutron star (around $1.25 M_{\odot}$ ), relatively little energy is needed to eject the remainder of the star; the resulting supernova is therefore expected to be of relatively low energy $\left(\sim 10^{43} \mathrm{~J}\right)$ and may produce a neutron star with a relatively low kick velocity (unlike the case of Fe-core collapse; see $\S 8.6$ for further discussion). The supernova that produced the Crab supernova in 1054 may provide an example for this type of explosion (Nomoto et al. 1982).

It should also be noted that an e-capture supernova can also occur in an accreting binary, where an accreting ONeMg white dwarf passes the threshold for the e-capture instability (so-called accretion-induced collapse [AIC]), or in the case of the merger of two white dwarfs of various different types $(\mathrm{CO}+\mathrm{CO}$, $\mathrm{ONeMg}+\mathrm{He}, \mathrm{ONeMg}+\mathrm{CO}$ or even $\mathrm{ONeMg}+\mathrm{ONeMg}$ [if possible]; so-called merger-induced collapse ([MIC]). These channels could produce as many as $\sim 10 \%$ of all neutron stars.

\section{Magnetars}

If the collapse of a massive star produces a rapidly rotating, magnetic neutron star, a magnetar, the rapid spin-down of this magnetar could potentially power a very energetic explosion on timescales of days to weeks (this is the neutronstar analogue to the collapsar model). As this would allow the extraction of a large fraction of the rotational energy of a neutron star, this could produce an extremely energetic supernova (see, e.g., Kasen \& Bildsten 2010). 


\section{Black Holes}

\section{Prompt or Fallback Collapse}

The formation of a black hole is probably rather unspectacular if no rotation is involved. If a black hole forms promptly, essentially most of the star is expected to collapse into the black hole. If a weak outward going shock is formed initially, but does not succeed to eject the whole core, a black hole can form in a delayed manner by fallback of the non-ejected core. In the first case, no supernova is expected, while the latter may produce a faint supernova if some of the envelope can be ejected. As this is the expected fate for massive single stars that form black holes, this implies that as many as $10 \%$ of core collapses may not be accompanied by a bright supernova event.

\section{Collapsars and Jet-Driven Explosions}

On the other hand, in the collapse of a rapidly rotating core (i.e., in the collapsar model; $\S 6.6$ ), a large fraction of the binding energy of the compact object can be extracted driving the powerful explosions often associated with long-duration GRBs. Even without a GRB, such jet-driven explosions could produce very energetic explosions (i.e., hypernovae or broad-lined SNe Ic).

\section{Thermonuclear Explosions}

\section{Type Ia Supernovae}

The thermonucler runaway in a CO white dwarf as it approaches the Chandrasekhar mass is the standard model for SNe Ia (§ 2.2).

\section{He Detonations}

If a helium shell detonates in an accreting white dwarf, this can lead to a rather bright explosion and could easily be mistaken for a faint supernova (Nomoto 1982). Indeed, as such detonations produce elements similar to a SN Ia, they may resemble a SN Ia and are therefore now sometimes referred to as .SNe Ia (note the period). The main difference is that the underlying CO white dwarf is likely to survive. On the other hand, if the helium detonation drives a sufficiently strong shock into the CO core, this could trigger a second detonation near the core, a carbon detonation, which would then destroy the whole white dwarf and produce a SN Ia-like event. Such double-detonation models are an alternative scenario for SNe Ia; they have the additional advantage that they could also occur in $\mathrm{CO}$ white dwarfs below the Chandrasekhar mass, which are statistically much more common (see Woosley \& Weaver 1994; Fink et al. 2010).

\section{Pair-Instability Supernovae}

There is another type of thermonuclear runaway that can occur in very massive oxygen cores when the core temperature becomes high enough $\left(\gtrsim 10^{9} \mathrm{~K}\right)$ 
that electron-positron pairs form abundantly. This leads to the conversion of internal energy into the rest-mass energy of the pairs and reduces the central thermal pressure support. As such cores are radiation-pressure supported and are ony marginally stable to start out with, this decreases the average adiabatic index below the critical value of $4 / 3$, triggering a partial collapse of the core. This accelerates the rate of oxygen burning and leads to a thermonuclear runaway that in most cases is expected to completely destroy the star. This type of supernova requires a very massive helium core after $\mathrm{H}$-core burning $\left(\gtrsim 60 M_{\odot}\right)$ which corresponds to an initial main-sequence mass of $\sim 140 M_{\odot}$ (Heger \& Woosley 2002), if there is no mass loss. If the He core is too massive ( $\gtrsim 130 M_{\odot}$ corresponding to a main-sequence mass of $\sim 260 M_{\odot}$ ), the photodisintegration of heavy nuclei becomes important. This involves endothermic reactions that completely use up the energy produced by oxygen burning, preventing it from reversing the initial collapse and ultimately leading to a collapse of the whole core to a rather massive black hole.

Even though the physics of this explosion mechanism has been well understood for a long time (Rakavy et al. 1967), pair-instability supernovae have traditionally not been considered important at solar metallicity. Even if such massive progenitor stars were able to form, the wind mass loss on the main sequence would prevent the formation of a sufficiently massive He core. Langer et al. (2007) estimate that the maximum allowed metallicity for a pair-instability supernova is $1 / 3$ solar. A pair-instability supernova could be extremely bright as several $M_{\odot}$ (perhaps even more than $10 M_{\odot}$ ) of radioactive $\mathrm{Ni}$ can be produced in such an event.

At Population III metallicity, these could, in principle, be the dominant source of Fe. However, detailed nucleosynthesis models also predict a strong odd-even effect in the nuclear abundance pattern, where nuclei with even nuclear charge are much more abundant than those with odd charge (Heger \& Woosley 2002). This would produce a nucleosynthetic imprint that is not actually observed. This implies that pair-instability supernovae cannot be so common to make a major contribution to the global nucleosynthesis.

A good candidate for a pair-instability supernova is SN 2007bi (Gal-Yam et al. 2009), a SN Ic with an ejecta mass estimated as $\sim 100 M_{\odot}$ and a $\mathrm{Ni}$ mass $\gtrsim 3 M_{\odot}$. It also occurred in a small dwarf galaxy, consistent with a low-metallicity progenitor.

\subsection{Rotation and Magnetic Fields}

Massive stars tend to be rapid rotators. In order for rotation to be dynamically important, the rotation rate has to be quite close to critical rotation (i.e., close to break-up) which stars rarely achieve except sometimes in their outer layers. This is particularly true for stars in binaries which can be spun up by accretion from a companion star or by merging with a companion. Apart from changing the appearance of a star (e.g., due to rotational flattening causing a non-uniform surface temperature distribution), the main effect of rotation is 
to cause additional mixing within the star (e.g., due to baroclinic instabilities). This can mix nuclear-processed material to the surface, a tell-tale sign of rapid rotation. For single stars, rotation is most important on the main sequence, as massive stars are very efficient in losing their angular momentum, mainly in the form of stellar winds that carry away mass and angular momentum. In addition, it is now believed that, if a star is differentially rotating, even small seed magnetic fields are amplified causing efficient redistribution of angular momentum inside a star and driving the rotation profile towards solid-body rotation (Heger et al. 2005). This is the reason why the cores of most massive stars are unlikely to be rotating rapidly at the time of core collapse, consistent with the fact that newborn radio pulsars appear not to be rotating near breakup. Therefore the main effect of rotation in single stars is to increase the final mass of the H-exhausted core at the end of the main sequence. Effectively, after its main-sequence phase, a rapidly rotating star will behave similar to a more massive star that has lost a larger fraction of its envelope (i.e., has a larger ratio of core mass to total mass).

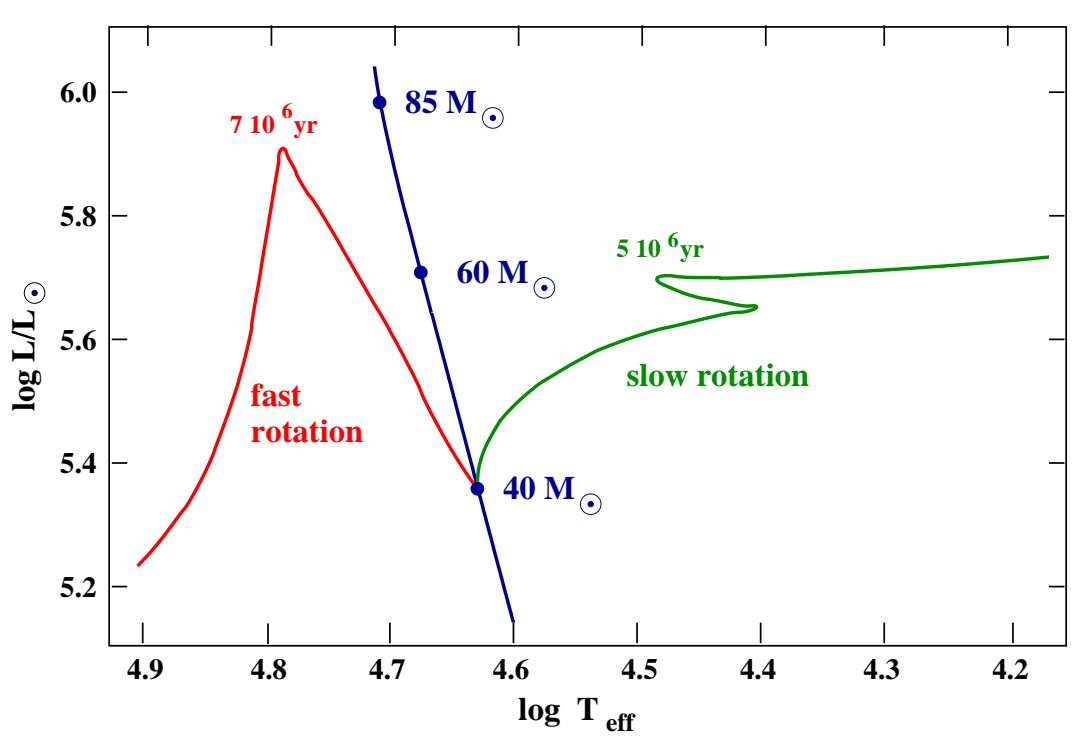

Fig. 16. Hertzsprung-Russell diagram illustrating the bifurcation of the evolution of a $40 M_{\odot}$ with slow and rapid rotation. Because of its almost homogeneous evolution, the rapidly rotating star evolves to the blue and avoids a red-supergiant phase. The blue curve shows the zero-age main sequence for massive stars with several masses indicated. (Figure adapted from Maeder [1987].) 
However, if a main-sequence star is rotating extremely rapidly, rotational mixing can be so efficient that it keeps the star almost homogeneously mixed; this will drastically change its evolution as such a star evolves towards the blue and is effectively converted into a Wolf-Ray-type star even without any mass loss (Maeder 1987). This is shown in Fig. 16, which shows the evolution of a $40 M_{\odot}$ with slow and with rapid rotation. Since such homogeneously evolving stars avoid a red-supergiant phase and the strong mass loss experienced in this phase, they may retain a much larger fraction of their initial angular momentum and may still have rapidly rotating cores at the time of the supernova. This makes them potential candidates for LGRBs in the collapsar model (see $\S 6.6)$.

\subsection{Metallicity}

The main effects of metallicity are to change the opacity and the mass-loss rate of a star. A lower metallicity will generally lower the opacity of the star (this is particularly true for low- and intermediate-mass stars), making them more luminous and shortening their lifetimes. One consequence of this is that it changes the thermodynamic structure of the core of a star. For example the core of a $5 M_{\odot}$ AGB star at a typical globular-cluster metallicity (with $Z \simeq 0.001$ ) is similar to the core of a $7 M_{\odot}$ star at solar metallicity. This implies that the initial-mass limits, shown in Table 2, have to be shifted accordingly.

Since heavy elements play an important role in driving mass loss from stars, the wind mass-loss rate is a strong function of metallicity (typically $\dot{M} \propto Z^{0.7}$ ). One implication of this is that lower- $Z$ stars do not only lose less mass, but also less angular momentum (see $\S 6.6$ ); this makes it more likely that a low- $Z$ star still has a rapidly rotating core at the time of explosion, which could explain why LGRBs appear to favour low- $Z$ environments. Also, because of the lower mass loss, the most massive stars may end with much more massive cores. This makes pair-instability supernovae more likely at low $Z$ as it is mainly determined by the core mass at the beginning of $\mathrm{O}$ burning.

Finally, for metal-free stars (so-called population III stars), the whole evolution of a massive star is drastically altered, as, e.g., on the main sequence nuclear burning has to take place via the pp cycle rather than the standard CNO cycle.

\subsection{Dynamical Interactions}

A significant fraction of stars are found in globular clusters which means that direct dynamical interactions can be important. This may be particularly true for massive stars that are often born in a cluster-type environment. Dynamical interactions (such as tidal captures, direct collisions, exchange interactions, etc.) are likely to enhance certain evolutionary channels (e.g., mergers of degenerate objects) and may even produce new types of stars that cannot be 
found elsewhere (not even in binaries). A particular example are runaway collisions at the centre of clusters that may produce much more massive stars than can ordinarily be formed; the Pistol star in the Quintuplet cluster near the Galactic centre, one of the most massive stars known in our Galaxy, is a potential candidate. The maximum mass that can be built up in this way is a strong function of the mass loss the object experiences between merger events, which will be strongly enhanced. With realistic mass loss prescriptions it presently seems difficult to form extremely massive stars at least at solar metallicity (see Gleebeck et al. 2009).

\section{Current Topics}

At the moment, we are in a golden epoch for supernova research as current all-sky surveys, such as the Palomar Transient Factory (PTF), Pan-STARRS and Skymapper, continue to discover more and more supernova types and sub-types. In this last section, I will discuss a selection of the topics that are considered 'hot' at the present time, a list that undoubtedly will grow in the near future.

\subsection{The Diversity of SN Ia Progenitors}

Some of the recent PTF supernovae have provided some important constraints on the progenitors of fairly normal SNe Ia, strongly suggesting that there is more than one progenitor channel: while PTF $11 \mathrm{kx}$ clearly must have occurred in a binary with a hydrogen-rich donor star (most likely a giant; Dilday et al. 2012), such a companion can firmly be ruled out in the case of SN 2011fe/PTF $11 \mathrm{kly}$ (Li et al. 2011). But there are even more unusual SNe Ia. The supernova SNLS-03D3bb provided the first example of a new class of SNe Ia where the exploding white dwarf appears to have a mass well in excess of the Chandrasekhar mass (once the mass in all the elements in the ejecta has been added up; Howell et al. 2006). This is possible in principle if the white dwarf is rotating rapidly as this can increase the critical mass up to $2 M_{\odot}$ and above (e.g. Yoon \& Langer 2005a). Alternatively, these "super-Chandrasekhar" SNe Ia could be the result of the direct dynamical merger of two rather massive $\mathrm{CO}$ white dwarfs.

\subsection{Superluminous Supernovae}

One of the major recent surprises has been the discovery of a class of unusually luminous supernovae. The first one of these reported was SN 2006gy which had a peak absolute magnitude of -22 , much brighter than a typical SN Ia with a peak magnitude of -19 . There is now a whole class of such supernovae, most of them hydrogen-free, reaching peak magnitudes of almost -23 
(see, e.g., Quimby et al. 2011). One early suggestion was that their lightcurves could be powered by the decay of several solar masses of radioactive $\mathrm{Ni}$, as can be produced in a pair-instability supernova (see $\S 7.2$ ). However their late lightcurves do not show the expected exponential decay ruling out this possibility. It should be noted that a very luminous lightcurve does not necessarily imply a very energetic explosion as, in a typical explosion, only a small fraction of the kinetic energy in the explosion is radiated away in the first few years after a supernova. Indeed, if a supernova is surrounded by a very dense medium or a shell with a mass comparable to the ejecta mass, the ejecta can, in principle, be slowed down on a timescale of less than a year, rapidly converting kinetic energy into thermal energy and radiation, i.e., power the lightcurve by the interaction with the medium. Alternatively, such supernovae could be powered by a long-lived central engine, such as a magnetar (see $\S 7.2$ ). These superluminous supernovae, which one should be able to detect up to a large redshift $(z \gtrsim 4)$, must be very rare and therefore require very special circumstances. Probably only 1 in $10^{4}$ massive stars end their lives in a superluminous supernova.

\subsection{Supernovae with a Circumstellar Medium}

A significant fraction of supernovae (perhaps as many as $6 \%$ ) appear to explode surrounded by a dense medium, indicating that they ejected a large amount of mass very recently before the explosion. These are generally classified as IIn or IIa supernovae (see $\S 3.2$ ) and may include both core-collapse and thermonuclear explosions. More surprisingly, there may even be some evidence that some explode in a luminous-blue variable (LBV) phase (reminiscent of the great outburst of $\eta$ Carinae; e.g., SN 2005gj [Trundle et al. 2008]). The latter would be very surprising as this is not consistent with the theoretically expected evolution of very massive stars that are expected to encounter the LBV instability near the end or just after the main-sequence phase. At this stage, they are assumed to lose their H-rich envelopes in a series of LBV outbursts and spend most of their remaining evolution (at least several $10^{5} \mathrm{yr}$ ) as Wolf-Rayet stars. If single massive stars were able to explode in an LBV phase, all evolutionary models for massive stars existing today would have to be discarded. However, as most massive stars are in relatively close binaries, there may be a simple alternative solution. As discussed in $\S 7.1$, if a massive star that has left the main sequence before it accretes matter or merges with a massive companion star, its subsequent evolution can be drastically altered. In particular, it will spend most of its helium core-burning phase as a blue supergiant and may only encounter the LBV instability after this phase, when its remaining lifetime will typically be less than a few $10^{3} \mathrm{yr}$; such a star could easily still be in an LBV phase at the time of the explosion. 


\subsection{Supernovae in the Middle of Nowhere}

Traditionally, supernova searches have targeted large galaxies introducing some serious observational biases. This has changed somewhat with the recent advent of untargeted, all-sky surveys. Supernovae are now also regularly found in small galaxies and - more surprisingly - far away from any galaxy. These supernovae often have quite distinct properties. One example is SN 2005E, a faint Ib supernova in the halo of a nearby galaxy (Perets et al. 2010). This supernova is also very rich in Ca, making it a prototype for a new supernova class. Since the estimated ejecta mass is very small (only $\sim 0.3 M_{\odot}$ ), it is not even clear whether this is a proper supernova or related to explosive helium burning on a white dwarf (see $\S 7.2$ ). Alternatively, it could be a hybrid supernova, part core collapse, part thermonuclear. For example, the merger of an $\mathrm{ONeMg}$ white dwarf with a He white dwarf could lead to the collapse the $\mathrm{ONeMg}$ core to a neutron star (in an e-capture supernova; $\S 7.2$ ) which triggers explosive burning of the helium envelope which in turn powers the explosion. Even though this would technically be classified as a core-collapse supernovae, the explosion would be driven by the thermonuclear runaway.

A second example is PTF10ops, a subluminous SN Ia, that occurred in the middle of nowhere (Maguire et al. 2011). Even though clearly a SN Ia, it is an unusual one that does not fit the standard SN Ia peak magnitude lightcurve width relation.

Why are these supernovae so far away from a major galaxy? Just because these channels require 'old' progenitors? Or were the explosions delayed because they involved the merger of two white dwarfs where the delay time is determined by the gravitational radiation timescale that drives the system together? One should also consider the possibility that these events occur in globular clusters in the halo of galaxies, which for these supernovae would be undetectable, and that these unusual supernovae are somehow connected to their unusual dynamcial environment.

\subsection{Faint and Failed Supernovae}

Single massive stars ( $\left.\gtrsim 20-25 M_{\odot}\right)$ are expected to form black holes, either promptly or by fallback. These are unlikely to be associated with a bright supernova event. Perhaps in many cases there is no supernova at all (a 'failed' supernova). This means that a star, in most cases probably a Wolf-Rayet star, should suddenly disappear. While difficult to detect such 'disappearing stars', with modern large-scale surveys this is not impossible (see, e.g., Kochanek et al. 2008).

\subsection{Supernova Kicks}

Young, single radio pulsars are observed to have a large space motion relative to their parent populations which is best described by a Mawellian distribution 
with a velocity dispersion of $265 \mathrm{~km} \mathrm{~s}^{-1}$ (Hobbs et al. 2005). This implies that these single neutron stars must have received a large kick when they were born in a supernova, which provides an important clue to the supernova mechanism. At present, the most promising explanation for these kicks in the delayed neutrino-driven explosion scenario $(\S 2.1)$ is an instability in the accretion shock around a proto-neutron star, the so-called SASI instability ('standing accretion-shock instability'), which causes a wobbling of the core, imparting momentum in the process (see, e.g., Blondin \& Mezzacappa 2007; Foglizzo et al. 2007). In order for this instability to be able to grow sufficiently, the delay between the initial formation of the proto-neutron star and the initiation of a "successful" explosion has to be $\gtrsim 500 \mathrm{~ms}$ (i.e. 100s of dynamical times), consistent with the most promising models of Fe core collapse to date. On the other hand, in the case of an e-capture supernova ( $\S 7.2$ ), where the binding energy of the inner part of the ejecta is very small, the explosion is expected to occur with a much shorter delay (e.g., Kitaura et al. 2006), suggesting that this will produce at best a moderate kick. Since e-capture supernovae are more likely to occur in binary systems, this could explain why neutron stars in close binary sometimes appear to have received a much smaller kick than the majority of their single counterparts (Podsiadlowski et al. 2004).

\section{References}

1. Arnet Arnett, D.: Astrophys. J., 253, 785 (1982)

2. Baade, W., Zwicky, F.: Phys. Rev., 4676 (1934)

3. Berger, E., et al.: Astrophys. J., 743, 204 (2011)

4. Blandford, R.D., Znajek, R.L.: Month. Not. R. Astron. Soc., 179, 433 (1977)

5. Blondin, J.M., Mezzacappa, A.: Nature, 445, 58 (2007)

6. Burrows, C.J., et al.: Astrophys. J., 452, 680 (1995)

7. Cappellaro, E., Turatto, M.: 1997, in Thermonuclear Supernovae, ed. RuizLapuente et al. (Kluwer, Dordrecht), p. 77

8. Cucchiara, A., et al.: Astrophys. J., 736, 7 (2011)

9. Brown, G.E., Heger, A., Langer, N., Lee, C.-H., Wellstein, S., Bethe, H.: New Astron., 6, 457 (2001)

10. Detmers, R.G., Langer, N., Podsiadlowski, Ph., Izzard, R.G.: Astron. E Astrophys., 484, 831 (2008)

11. Dilday, B., et al.: submitted (2012)

12. Filippenko, A.V.: Ann. Rev. Astron. Astrophys., 35, 309 (1997)

13. Fink, M., et al.: Astron. \& Astrophys., 514, 53 (2010)

14. Foglizzo, T., Galletti, P., Scheck, L., Janka, H.-T.: Astrophys. J., 654, 1006 (2007)

15. Frail, D.A., et al.: Astrophys. J., 562, 55 (2001)

16. Fryer, C.L., et al.: Publ. Astron. Soc. Pacific, 119, 1211 (2007)

17. Fryer, C.L., Kalogera, V.: Astrophys. J., 554, 548 (2001)

18. Gal-Yam, A., et al.: Nature, 462, 624 (2009)

19. Gamow, G., Schoenberg, M.: Phys. Rev., 59, 539 (1941) 
20. Gleebeek, E., Gaburov, E., de Mink, S.E., Pols, O.R., Portegies Zwart, S.F.: Astron. \& Astrophys., 497, 255 (2009)

21. Hachinger, S., Mazzali, P.A., Taubenberger, S., Hillebrandt, W., Nomoto, K., Sauer, D.N.: arXiv:1201.1506 (2012)

22. Hachisu, I., Kato, M., Nomoto, K., Umeda, H.: Astrophys. J., 519, 314 (1999)

23. Han, Z., Podsiadlowski, Ph., Eggleton, P.P.: Month. Not. R. Astron. Soc., 272, $800(1995)$

24. Heger, A., Woosley, S.E.: Astrophys. J., 567, 532 (2002)

25. Heger A., Woosley, S.E., Spruit H.: Astrophys. J., 626, 350 (2005)

26. Hillebrandt, W., Meyer, F.: Astron. \&s Astrophys., 219, 3 (1989)

27. Hobbs, G., Lorimer, D.R., Lyne, A.G., Kramer, M.: Month. Not. R. Astron. Soc., 360, 974 (2005)

28. Howell, D.A., et al.: Nature, 443, 308 (2006)

29. Hsu, J.J.L.: PhD Thesis (M.I.T.) (1991)

30. Iben, I., Jr., Tutukov, A.V.: Astrophys. J. Suppl., 54, 335 (1984)

31. Ivanova, N., Podsiadlowski, Ph.: in From Twilight to Highlight: the Physics of Supernovae, ed. W. Hillebrandt, B. Leibundgut (Springer, Berlin), p. 19 (2003)

32. Iwamoto, K., et al.: Nature, 395, 672 (1998)

33. Izzard, R.G., Ramirez-Ruiz, E., Tout, C.A.: Month. Not. R. Astron. Soc., 348, $1215(2004)$

34. Kasen, D., Bildsten, L.: Astrophys. J., 717, 245 (2010)

35. Kitaura, F.S., Janka, H.-Th., Hillebrandt, W.: Astron. ES Astrophys., 450, 345 (2006)

36. Klebesadel, R.W.; Strong, I.B.; Olson, R.A.: Astrophys. J., 182, 85 (1973)

37. Kochanek, C.S., et al.: Astrophys. J., 684, 1336 (2008)

38. Langer, N., Norman, C.A., de Koter, A., Vink, J.S., Cantiello, M., Yoon, S.-C.: Astron. \& Astrophys., 475, 19 (2007)

39. Levesque, E.M., Kewley, L.J., Graham, J.F., Fruchter, A.S.: Astrophys. J., 712, $26(2010)$

40. Li, W., et al.: Nature, 480, 348 (2011)

41. Maeder, A.: Astron. ES Astrophys., 178159 (1987)

42. Maguire, K., et al.: Month. Not. R. Astron. Soc., 418, 747 (2011)

43. Maund, J.R., Smartt, S.J., Kudritzki, R.P., Podsiadlowski, Ph.; Gilmore, G.F.: Nature, 427, 129 (2004)

44. Mazzali, P.A., Nomoto, K., Cappellaro, E., Nakamura, T., Umeda, H., Iwamoto, K.: Astrophys. J., 547, 988 (2001)

45. Meszaros, P., Rees, M.J.: Astrophys. J., 405, 278 (1993)

46. Metzger, B.D., Giannios, D., Thompson, T.A., Bucciantini, N., Quataert, E.: Month. Not. R. Astron. Soc., 413, 2031 (2011)

47. Morris, T., Podsiadlowski, Ph.: Science, 315, 1103 (2007)

48. Nelemans, G., Yungelson, L.R., Portegies Zwart, S.F., Verbunt, F.: Astron. $\&$ Astrophys., 365, 491 (2001)

49. Nomoto, K.: 1982, Astrophys. J., 253, 798

50. Nomoto, K.: Astrophys. J., 277, 791 (1984)

51. Nomoto, K., Iben, I., Jr.: Astrophys. J., 297, 531 (1985)

52. Nomoto, K., Sugimoto, D., Sparks, W.M., Fesen, R.A., Gull, T.R., Miyaji, S.: Nature, 299, 803 (1982)

53. Paczyński B.: in Structure and Evolution in Close Binary Systems, ed. P.P. Eggleton, S. Mitton, J. Whelan (Dordrecht, Reidel), p. 75 (1976) 
54. Perets, H.B., et al.: Nature, 465, 322 (2010)

55. Perlmutter, S., et al.: Astrophys. J., 517, 565 (1999)

56. Phillips, M.M.: Astrophys. J., 413, L105 (1993)

57. Podsiadlowski, Ph., Dewi, J.D.M., Lesaffre, P., Miller, J.C., Newton, W.G., Stone, J.R.: Month. Not. R. Astron. Soc., 361, 1243 (2005)

58. Podsiadlowski, Ph., Ivanova, N., Justham, S., Rappaport, S.: Month. Not. R. Astron. Soc., 406, 840 (2010)

59. Podsiadlowski, Ph., Joss, P.C.: Nature, 338, 401 (1989)

60. Podsiadlowski, Ph., Joss, P.C., Hsu, J.J.L.: Astrophys. J., 391, 245 (1992)

61. Podsiadlowski, Ph., Joss, P.C., Rappaport, S.: Astron. \& Astrophys., 227, L9 (1990)

62. Podsiadlowski, Ph., Langer, N., Poelarends, A.J.T., Rappaport, S., Heger, A., Pfahl, E.: Astrophys. J., 612, 1044 (2004)

63. Podsiadlowski, Ph., Mazzali, P.A., Nomoto, K., Lazzati, D., Cappellaro, E.: Astrophys. J., 607, L17 (2004)

64. Quimby, R.M., et al.: Nature, 474, 487 (2011)

65. Rakavy, G., Shaviv, G., Zinamon, Z.: Astrophys. J., 150, 131 (1967)

66. Riess, A.G., et al.: Astron. J., 116, 1009 (1998)

67. Schawinski, K., et al.: Science, 321, 223 (2008)

68. Taylor, P.A., Miller, J.C., Podsiadlowski, Ph.: Month. Not. R. Astron. Soc., 410, 2385 (2011)

69. Timmes, F.X., Brown, Edward F., Truran, J.W.: Astrophys. J., 590, 83 (2003)

70. Toomre, A.: Astrophys. J., 139, 1217 (1964)

71. Trundle, C., Kotak, R., Vink, J.S., Meikle, W.P.S.: Astron. ES Astrophys., 483, L47 (2008)

72. van den Heuvel, E.P.J., Bhattacharya, D., Nomoto, K., Rappaport, S.: Astron. \&) Astrophys., 262, 97 (1992)

73. Webbink, R.F.: Astrophys. J., 277, 355 (1984)

74. Whelan, J., Iben, I., Jr.: Astrophys. J., 186, 1007 (1973)

75. Woosley, S.E.: Astrophys. J., 405, 273 (1993)

76. Woosley, S.E., Heger, A.: Astrophys. J., 637, 914 (2006)

77. Woosley, S.E., Weaver, T.A.: Astrophys. J., 423, 371 (1994)

78. Yoon, S.-C., Langer, N.: Astron. \&6 Astrophys., 419, 623 (2004)

79. Yoon, S.-C., Langer, N.: Astron. $\&$ Astrophys., 435, 967 (2005a)

80. Yoon, S.-C., Langer, N.: Astron. \& Astrophys., 443, 643 (2005b)

81. Yoon, S.-C., Langer, N., Norman, C.: Astron. ES Astrophys., 460, 199 (2006)

82. Yoon, S.-C., Podsiadlowski, Ph., Rosswog, S.: Month. Not. R. Astron. Soc., 390, 933 (2007)

83. Yungelson, L.R., Livio, M., Tutukov, A.V., Saffer, R.: Astrophys. J., 420, 336 (1994) 